\title{
Transcriptomic and biochemical analysis of upland cotton (Gossypium hirsutum) and a chromosome segment substitution line from $G$. hirsutum $\times$ G. barbadense in response to Verticillium dahliae infection
}

\author{
Peng-tao Li ${ }^{1,2+}$, Md. Harun or Rashid ${ }^{1 \dagger}$, Ting-ting Chen ${ }^{1,3+}$, Quan-wei Lu², Qun Ge', Wan-kui Gong ${ }^{1}$, Ai-ying Liu',
}

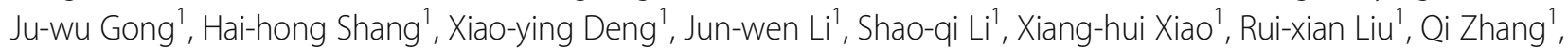
Li Duan' ${ }^{1}$ Xian-yan Zou' ${ }^{1}$ Zhen Zhang ${ }^{1}$, Xiao Jiang ${ }^{1}$, Ya Zhang ${ }^{2}$, Ren-hai Peng ${ }^{2}$, Yu-zhen Shi ${ }^{*{ }^{*}}$ and You-lu Yuan ${ }^{1 *}$ (D)

\begin{abstract}
Background: Verticillium wilt (VW), also known as "cotton cancer," is one of the most destructive diseases in global cotton production that seriously impacts fiber yield and quality. Despite numerous attempts, little significant progress has been made in improving the VW resistance of upland cotton. The development of chromosome segment substitution lines (CSSLs) from Gossypium hirsutum $\times$ G. barbadense has emerged as a means of simultaneously developing new cotton varieties with high-yield, superior fiber, and resistance to $\mathrm{WW}$.

Results: In this study, WW-resistant investigations were first conducted in an artificial greenhouse, a natural field, and diseased nursery conditions, resulting in the identification of one stably WW-resistant CSSL, MBI8255, and one VW-susceptible G. hirsutum, CCRI36, which were subsequently subjected to biochemical tests and transcriptome sequencing during V991 infection (0, 1, and 2 days after inoculation). Eighteen root samples with three replications were collected to perform multiple comparisons of enzyme activity and biochemical substance contents. The findings indicated that W resistance was positively correlated with peroxidase and polyphenol oxidase activity, but negatively correlated with malondialdehyde content. Additionally, RNA sequencing was used for the same root samples, resulting in a total of 77,412 genes, of which 23,180 differentially expressed genes were identified from multiple comparisons between samples. After Gene Ontology and Kyoto Encyclopedia of Genes and Genomes enrichment analysis on the expression profiles identified using Short Time-series Expression Miner, we found that the metabolic process in the biological process, as well as the pathways of phenylpropanoid biosynthesis and plant hormone signal transduction, participated significantly in the response to WW. Gene functional annotation and expression quantity analysis indicated the important roles of the phenylpropanoid metabolic pathway and oxidation-reduction process in response to $\mathrm{WW}$, which also provided plenty of candidate genes related to plant resistance.

(Continued on next page)
\end{abstract}

\footnotetext{
*Correspondence: shiyuzhen@caas.cn; youluyuan@hotmail.com

${ }^{\dagger}$ Peng-tao Li, Md. Harun Rashid and Ting-ting Chen contributed equally to this work.

${ }^{1}$ State Key Laboratory of Cotton Biology, Key Laboratory of Biological and Genetic Breeding of Cotton, The Ministry of Agriculture, Institute of Cotton Research, Chinese Academy of Agricultural Science, Anyang 455000, Henan, China

Full list of author information is available at the end of the article
}

(c) The Author(s). 2019 Open Access This article is distributed under the terms of the Creative Commons Attribution 4.0 International License (http://creativecommons.org/licenses/by/4.0/), which permits unrestricted use, distribution, and reproduction in any medium, provided you give appropriate credit to the original author(s) and the source, provide a link to the Creative Commons license, and indicate if changes were made. The Creative Commons Public Domain Dedication waiver (http://creativecommons.org/publicdomain/zero/1.0/) applies to the data made available in this article, unless otherwise stated. 
(Continued from previous page)

Conclusions: This study concentrates on the preliminary response to V991 infection by comparing the WW-resistant CSSL and its WW-susceptible recurrent parent. Not only do our findings facilitate the culturing of new resistant varieties with high yield and superior performance, but they also broaden our understanding of the mechanisms of cotton resistance to $\mathrm{W}$.

Keywords: Gossypium hirsutum, Chromosome segment substitution lines, Verticillium wilt, Biochemical tests, Transcriptome analysis

\section{Background}

Cotton, as one of the most important commercial crops, is the most widely cultivated fiber plant globally, and it is of great economic and social significance [1]. Despite being comprised of 46 diploid $(2 \mathrm{n}=2 \mathrm{x}=26)$ and 5 allotetraploid $(2 \mathrm{n}=4 \mathrm{x}=52)$ species, only 4 Gossypium species are widely cultivated, namely, G. arboreum, G. herbaceum, G. hirsutum, and G. barbadense [2]. As the major allotetraploid cotton species, upland cotton (G. hirsutum) and Sea Island cotton (G. barbadense) contribute more than 95\% fiber capacity, and they originated from a hybridization event between $G$. arboreum $\left(\mathrm{A}_{\mathrm{t}}\right.$ sub-genome) and $G$. raimondii ( $D_{t}$ sub-genome) 1-2 million years ago [3]. Verticillium wilt (VW), a representative vascular disease mainly caused by the infection of Verticillium dahliae Kleb., and the soil-borne fungus can cause leaf yellowing, wilt, defoliation, and even death [4]. Cotton VW is the most destructive disease, presently tremendously affecting cotton yield and fiber performance $[5,6]$.

Initially described on upland cotton in 1914 in the USA, VW was subsequently introduced into China via the importation of American cotton species, resulting in substantial cotton yield losses [7]. Verticillium dahliae can cause wilt disease on more than 200 plant species, indicating its extensive range of host plants $[8,9]$. The microsclerotia (melanized survival structures) formed by the soil-borne pathogen can survive in the soil for over 10 years, with germination being triggered by root exudate signals $[10,11]$. Unfortunately, there are no appropriate commercial fungicides available for G. hirsutum, while G. barbadense, despite possessing innate resistance to VW and premium fiber quality, experiences low-fiber productivity upon infection [12]. The above-mentioned conditions reduce the effectiveness of common cultivation practices, such as crop rotation, chemical fumigation, and soil conditioning [13]. Thus, the development of new cotton cultivars possessing VW resistance by means of traditional breeding and transgenic strategies is the most practical and cost effective method to manage cotton VW [14-16]. Given the increasing human population and diminishing arable land, the development of novel varieties harboring high yield, superior fiber quality, and VW resistance remains a significant topic in cotton breeding. In response, chromosome segment substitution lines (CSSLs) have emerged as a means of combining the advantages of both upland and Sea Island cotton. Through conventional breeding methods, such as hybridization, backcrossing, selfing, and marker assisted-selection (MAS), CSSLs were developed as the ideal materials for further genome research and crop improvement. CSSLs have been preferentially applied to the quantitative trait locus (QTL) mapping for essential traits, such as yield, quality, disease resistance, and stress tolerance in tomato [17], wheat [18], rice [19, 20], and cotton [21-26].

Plants have evolved two-layered immune mechanisms to protect themselves against various pathogens with different invasion strategies. The first-layer defense principally occurs on the extracellular surface of the host cell, where the pattern recognition receptors (PRRs) detect the pathogen-associated molecular patterns (PAMPs), further causing PAMP-triggered immunity (PTI) by the stimulation of PRRs [27, 28]. Although most pathogens are blocked by the basal immune response, some are able to successfully invade and suppress PTI through the deliverance of pathogenic effector proteins into the host cells via a type III secretion system (TTSS) [29]. Subsequently, the specific resistance (R) genes encoding nucleotide-binding site (NBS) and leucine-rich repeat (LRR) domains recognize these pathogen effectors, ultimately activating the second-layer defense mechanism, namely, effector-triggered immunity (ETI) [27, 28]. Plant defense reactions in response to diverse pathogens are triggered along with the recognition of the PRRs and $R$ genes, further causing a localized activation of programmed cell death (PCD), also known as the hypersensitive response (HR) [30,31]. Plenty of $R$ proteins in plants share two typical domains: a nucleotide-binding site (NBS) and a C-terminal leucine-rich repeat (LRR) region [32]. The former constitutes part of the nucleotide-binding (NB)-ARC adaptor shared by the apoptotic protease-activating factor 1 (APAF-1), R proteins, and Caenorhabditis elegans homolog domain 4 (CED-4) [33], while the latter proteins play a central role in the biological processes of plant growth and development, including the immune response [34]. Based on the 
$\mathrm{N}$-terminal domain categories, plant NBS-LRR proteins can be classified into toll/interleukin-1 receptors (TIR) and coiled-coil (CC), respectively referred to as TIR-NBS-LRR and CC-NBS-LRR $[35,36]$.

Along with the rapid development of next-generation sequencing technologies, diploid and allotetraploid Gossypium species have been successfully sequenced [3742], which has improved our understanding of cotton polyploid properties and provided a solid foundation for further functional genomics. Transcriptome sequencing, also known as RNA-Seq, concentrates on gene expression and transcriptional regulation, making it suitable for revealing the molecular mechanisms of particular biological processes. RNA-Seq has been widely applied to identify the key signaling pathways or resistant genes in response to VW not only in cotton [12, 43], but also in Nicotiana benthamiana [44], smoke tree [45], tomato [46], sunflower [47], and olive [48]. Furthermore, the phenylalanine pathway, which includes lignin and flavonoid biosynthesis, has been reported to be associated with VW resistance and is similarly regulated by some enzyme activities and biochemical substances, including polyphenol oxidase (PPO), phenylalanine ammonia lyase (PAL), peroxidase (POD), superoxide dismutase (SOD), and malondialdehyde (MAD) [12, 43].

In the present study, VW resistance investigations in artificial greenhouse, natural field, and disease nursery conditions were first conducted, and MBI8255, the VW-resistant CSSL, and CCRI36, the VW-susceptible recurrent parent, were subsequently used to perform biochemical tests during the preliminary stages of the V991 infection process $(0,1$, and 2 days after inoculation, DAI), which included assessing the activities of CAT, POD, SOD, PAL, and PPO, and the contents of MDA, proline, soluble sugar, and soluble protein. Additionally, RNA-Seq was carried out on the same samples, and plenty of differentially expressed genes (DEGs) relevant to VW resistance were identified, which were subjected to Gene Ontology (GO) and Kyoto Encyclopedia of Genes and Genomes (KEGG) enrichment analysis. This report not only identified various candidate genes associated with VW resistance, but also identified the key signaling pathways in response to V991 infection, which should elucidate the molecular mechanisms of cotton VW resistance and provide a solid foundation for cotton breeding and genomics research.

\section{Results}

Phenotypic disease index (DI) in the greenhouse tests

In order to evaluate the resistance to VW caused by V991, greenhouse tests with three replications of MBI8255, CCRI36, and two controls (Zhongzhimian2 and Jimian11) were conducted in 2015, with the phenotyping characterizations respectively collected at 15 and
30 DAI (Table 1). At 15 DAI, higher DI values were recorded in Jimian11 (27.11\%) and CCRI36 (15.38\%), which are susceptible to VW, while lower DI values were found in the resistant Zhongzhimian2 (1.10\%) and MBI8255 (3.26\%). Based on Least Significant Difference (LSD) tests at $P=0.05$, no significant difference was observed between the DI values of the CSSL MBI8255 and resistant control Zhongzhimian2, which were significantly lower than those of the recurrent parent CCRI36 and the susceptible control Jimian11 (Fig. 1). Furthermore, a similar phenomenon arose in the DI values of the four materials at 30 DAI. The highest DI value was observed in Jimian11 (51.81\%), while the lowest DI value was recorded in Zhongzhimian2 (16.18\%). The second highest and lowest DI values were separately recorded in CCRI36 (45.67\%) and MBI8255 (20.38\%). Based on the LSD test, significant differences were found not only between MBI8266 and Zhongzhimian2, but also between CCRI36 and Jimian11, while the DI values of the former two were significantly lower than those of the latter two.

\section{Phenotypic DI in the natural field and disease nursery tests}

To investigate VW resistance in the natural environment, natural field tests of MBI8255, CCRI36, and two controls (Zhongzhimian2 and Jimian11) were respectively performed in Anyang (Henan Province) with one replication and in Shihezi (Xinjiang Province) with one replication in 2015, with the phenotypic identifications being separately carried out at the flowering and maturity stages (Table 1). In the field tests, the DI values of the two controls, Jimian11 and Zhongzhimian2, varied from 44.74 and $5.67 \%$ at the flowering stage to 53.41 and $17.05 \%$ at the maturity stage in Anyang, while those of MBI8255 and CCRI36 varied from 6.73 and $27.89 \%$ at the flowering stage in Anyang to 23.91 and $48.16 \%$, respectively. With regards to the field tests in Xinjiang, the DI values of the VW-resistant Zhongzhimian2 and MBI8255 varied from $1.12 \%$ and 0 to 27.49 and $29.69 \%$, and those of the VW-susceptible Jimian11 and CCRI36 varied from 8.7 and $5.84 \%$ to 53.52 and $41.59 \%$, respectively.

Disease nursery tests were conducted to evaluate the DI values of MBI8255, CCRI36, and Jimian11 in Kaifeng (Henan Province) with one replication, and only the maturity stage was chosen to determine the phenotypic characteristics, ultimately resulting in DI values of 6.82 , 33.33 , and $56.77 \%$, respectively.

\section{Biochemical responses to V991 infection in MBI8255 and CCRI36}

To explore the relationships between cotton VW and the specific signaling pathways or biochemical substances, biochemical tests of MBI8255 and CCRI36 in 
Table 1 Descriptive statistics of DI in greenhouse, field and disease nursery tests

\begin{tabular}{|c|c|c|c|c|c|c|}
\hline Test & Phenotype & Env & MBI8255 & CCRI36 & Jimian11 & Zhongzhimian2 \\
\hline \multirow[t]{2}{*}{ Greenhouse } & $\mathrm{Dl}(\%)$ & 15DIA & 3.26 & 15.38 & 27.11 & 1.1 \\
\hline & & 30DIA & 20.38 & 45.67 & 51.81 & 16.18 \\
\hline \multirow[t]{4}{*}{ Field } & $\mathrm{Dl}(\%)$ & AYF15 & 6.73 & 27.89 & 44.74 & 5.67 \\
\hline & & AYM15 & 23.91 & 48.16 & 53.41 & 17.05 \\
\hline & & XJF15 & 0 & 5.84 & 8.7 & 1.12 \\
\hline & & XJM15 & 29.69 & 41.59 & 53.52 & 27.49 \\
\hline Disease nursery & Dl(\%) & KFM15 & 6.82 & 33.33 & 56.77 & \\
\hline
\end{tabular}

response to V991 infection were conducted on the enzyme activity or biochemical substances in the roots at 0 , 1, and 2 DAI. Three protective enzymes, namely, CAT, POD, and SOD, were chosen to analyze their activity changes during the process of V991 infection. The results are shown in Fig. 2a-c. CAT activity in the roots of CCRI36 gradually decreased from 0 DAI $(26.42 \mathrm{U} / \mathrm{g})$ to 2 DAI $(13.28 \mathrm{U} / \mathrm{g})$, but exhibited little change in the root samples of MBI8255, in which the highest and lowest activities occurred at 0 DAI $(19.24 \mathrm{U} / \mathrm{g})$ and 1 DAI (17.62 $\mathrm{U} / \mathrm{g})$. The POD activity first increased from 0 DAI $(1728 \mathrm{U} / \mathrm{g})$ to $1 \mathrm{DAI}(2389.33 \mathrm{U} / \mathrm{g})$, and then decreased at 2 DAI $(2109.33 \mathrm{U} / \mathrm{g})$ in the roots of CCRI36, while an opposite trend was observed in POD activity in the MBI8255 root samples, which first declined from 0 DAI $(2957.33 \mathrm{U} / \mathrm{g})$ to $1 \mathrm{DAI}(2189.33 \mathrm{U} / \mathrm{g})$ and then peaked at 2 DAI $(3528 \mathrm{U} / \mathrm{g})$. In regard to root SOD, its activity in CCRI36 gradually increased from 0 DAI $(289.49 \mathrm{U} / \mathrm{g})$ to 2 DAI $(334.37 \mathrm{U} / \mathrm{g})$, with the roots of MBI8255 exhibiting the highest and lowest SOD activity at 1 DAI (295.18 U/g) and 2 DAI (350.17 U/g), respectively.

Two defense enzymes relevant to VW resistance, namely, PAL and PPO, were also selected to investigate the activity changes following V991 infection (Fig. 2d-e). Only the PAL and PPO changes in the roots of CCRI36 showed a similar trend, first increasing from $3.94 \mathrm{U} / \mathrm{g}$

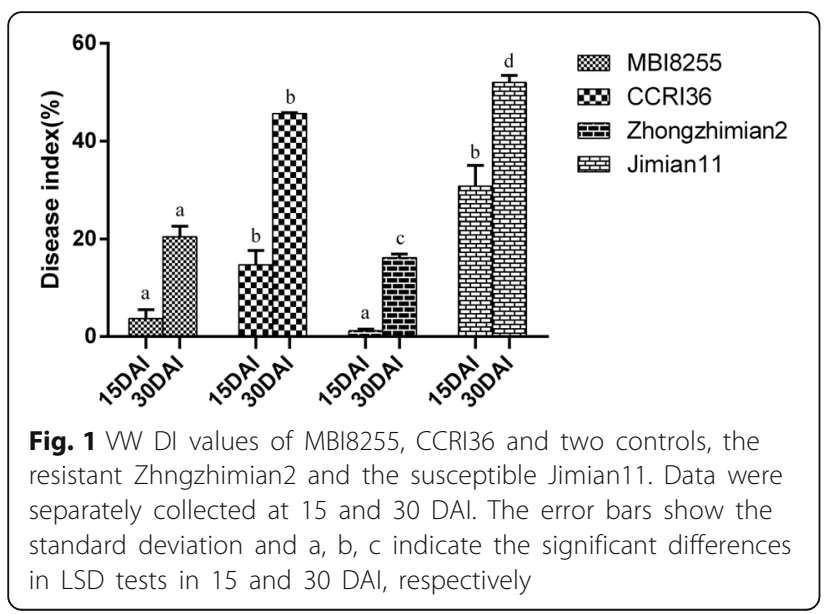

and $28.32 \mathrm{U} / \mathrm{g}$ at $0 \mathrm{DAI}$ to $5.61 \mathrm{U} / \mathrm{g}$ and $39.83 \mathrm{U} / \mathrm{g}$ at 1 DAI, and then declining to $5.45 \mathrm{U} / \mathrm{g}$ and $38.4 \mathrm{U} / \mathrm{g}$ at 2 DAI, respectively. The high PAL activity of the roots of MBI8255 at 0 DAI $(5.92 \mathrm{U} / \mathrm{g})$ dropped to the lowest value at $1 \mathrm{DAI}(5.29 \mathrm{U} / \mathrm{g})$, and then rose to $5.55 \mathrm{U} / \mathrm{g}$ at 2 DAI, while the PPO activity in the roots of MBI8255 rose from the lowest value of $33.12 \mathrm{U} / \mathrm{g}$ at $0 \mathrm{DAI}$ to the highest value of $48.48 \mathrm{U} / \mathrm{g}$ at $2 \mathrm{DAI}$.

Similarly, the contents of MDA, proline, soluble sugar, and soluble protein were assessed in the roots during V991 infection (Fig. 2f-i). Similar variation patterns were observed in both MDA and proline contents in the roots of the two lines, which showed a gradual decrease in CCRI36, whereas MBI8255 initially rose and then declined. The highest and lowest MDA contents in CCRI36 were $6 \mathrm{nmol} / \mathrm{g}$ at $0 \mathrm{DAI}$ and $4.29 \mathrm{nmol} / \mathrm{g}$ at 2 DAI, respectively, while those in MBI8255 were 15.43 $\mathrm{nmol} / \mathrm{g}$ at $1 \mathrm{DAI}$ and $9.43 \mathrm{nmol} / \mathrm{g}$ at $0 \mathrm{DAI}$, respectively. The maximum and minimum proline values in CCRI36 were $8.87 \mu \mathrm{g} / \mathrm{g}$ at $0 \mathrm{DAI}$ and $7.73 \mu \mathrm{g} / \mathrm{g}$ at $2 \mathrm{DAI}$, respectively, while those in MBI8255 were $8.65 \mu \mathrm{g} / \mathrm{g}$ at $1 \mathrm{DAI}$ and $6.78 \mu \mathrm{g} / \mathrm{g}$ at $2 \mathrm{DAI}$, respectively. The soluble sugar content of the roots of CCRI36 increased from $5.76 \mathrm{mg} /$ $\mathrm{g}$ at $0 \mathrm{DAI}$ to $6.59 \mathrm{mg} / \mathrm{g}$ at $1 \mathrm{DAI}$, and then declined to $5.75 \mathrm{mg} / \mathrm{g}$ at $2 \mathrm{DAI}$. The soluble sugar content in MBI8255 showed a gradual increase from $4.07 \mathrm{mg} / \mathrm{g}$ at 0 DAI to $6 \mathrm{mg} / \mathrm{g}$ at $2 \mathrm{DAI}$. The soluble protein content in the roots of CCRI36 first rose from $1.31 \mathrm{mg} / \mathrm{g}$ at $0 \mathrm{DAI}$ to $1.55 \mathrm{mg} / \mathrm{g}$ at $1 \mathrm{DAI}$ and then declined to $1.50 \mathrm{mg} / \mathrm{g}$ at 2 DAI, while MBI8255 showed an opposite pattern, decreasing from $1.79 \mathrm{mg} / \mathrm{g}$ at 0 DAI to $1.54 \mathrm{mg} / \mathrm{g}$ and then increasing to $1.63 \mathrm{mg} / \mathrm{g}$ at $2 \mathrm{DAI}$.

\section{Transcriptome sequencing and alignment to the $G$. hirsutum genome}

In this study, 18 RNA-Seq libraries were constructed from root samples infected by V991, which were collected at 0, 1, and 2 DAI in MBI8255 and CCRI36 with three biological replications, with the aim of systematically identifying the key genes or pathways affecting cotton resistance to VW. In total, 922.867 million raw reads were obtained, which were filtered for low-quality reads, 

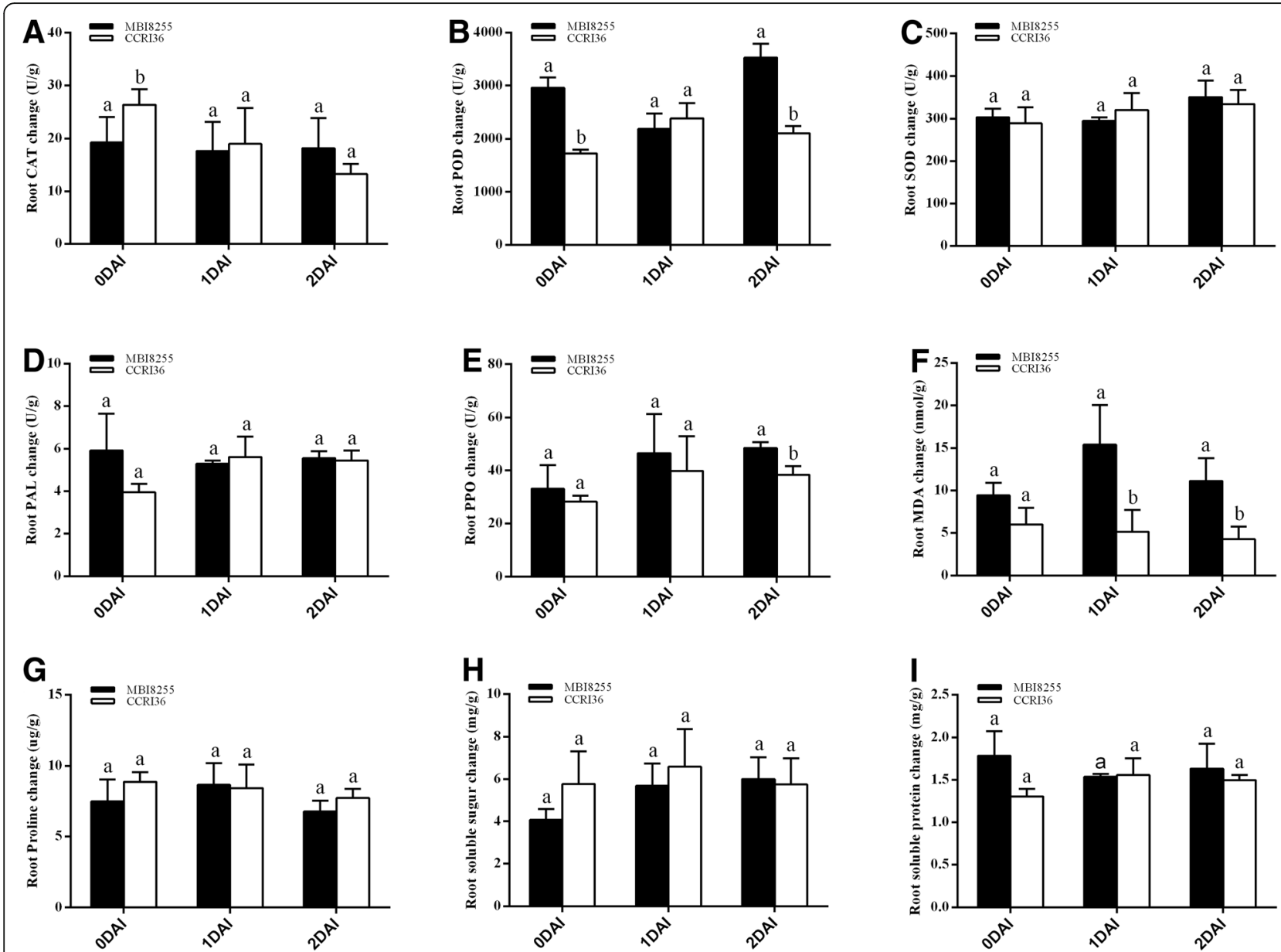

Fig. 2 Biochemistry analysis of MBI8255 and CCRI36 in response to V991 infection. a-c: the activity changes of 3 protective enzymes relevant to WW resistance (CAT, POD and SOD) in root at 0,1 and 2 DAl infected by V991. d-e: the activity changes of 2 defensive enzymes relevant to WW resistance (PAL and PPO) in root at 0, 1 and 2 DAl infected by V991. f-i: the content changes of 4 physiochemical substances relevant to WW resistance (MDA, Proline, soluble sugar and soluble protein) in root at 0, 1 and 2 DAl infected by V991. Three biological replications were performed and the error bars represent the standard deviation

resulting in 908.547 million clean reads (approximately $136.27 \mathrm{~Gb}$ data) with an average of $7.57 \mathrm{G}$ per library (Table 2). Over $91.38 \%$ of the Q30 values and no less than $43.44 \%$ of the GC content were calculated from the RNA-Seq results, of which the average values were 92.23 and $43.68 \%$, respectively. Subsequently, the clean reads were mapped to the G. hirsutum genome using TopHat2 software, and $87.69-91.23 \%$ of the clean data were successfully matched to the reference genome, of which $79.13-84.29 \%$ and $6.63-8.39 \%$ constituted unique and multiple reads, respectively. All of the above-mentioned results implied the reliability of our transcriptome results.

A total of 77,412 genes containing 6934 novel genes were found in our RNA-Seq results, of which the expression quantities were subsequently evaluated based on the Fragments Per Kilobase of transcript per Million mapped reads (FPKM) value. Pearson Correlation
Coefficient (PCC) analysis was used to calculate the correlations between the 18 experimental samples, approximately resulting in more than $93.9 \%$ similarity in the gene expressions of each sample based on three replications; however, exceptions were observed for 82.5 and $84 \%$ of similarities separately identified between CCRI36-2 III and CCRI36-2 I/CCRI36-2 II (Fig. 3). We found $92.4-97 \%$ similarity between the gene profiles of CCRI36 and MBI8255 at 0 DAI, whereas the similarities were $85.7-91.6 \%$ and $89-91.5 \%$, respectively, at 1 and 2 DAI, which suggested that the two lines at the same stages of V991 infection exhibited a similar gene expression pattern even though the similarities at 1 and 2 DAI were lower than those at 0 DAI. A gradual decrease in expressed gene similarity was observed between CCRI36-0 and CCRI36-1/CCRI36-2, first declining from 0 DAI to $1 \mathrm{DAI}$ and then increasing at $2 \mathrm{DAI}$ in MBI8255. The differences between the two lines might 
Table 2 Throughput and quality of RNA-seq of the 18 libraries

\begin{tabular}{|c|c|c|c|c|c|c|c|c|}
\hline Libraries & $\begin{array}{l}\text { Raw } \\
\text { reads }\end{array}$ & $\begin{array}{l}\text { Clean } \\
\text { reads }\end{array}$ & Clean bases & $\begin{array}{l}\text { Q30 } \\
(\%)\end{array}$ & $\begin{array}{l}\mathrm{GC} \\
(\%)\end{array}$ & Total match(\%) & $\begin{array}{l}\text { Multiple } \\
\text { match(\%) }\end{array}$ & $\begin{array}{l}\text { Unique } \\
\text { match(\%) }\end{array}$ \\
\hline CCRI36-0I & $56,789,392$ & $55,874,790$ & $8.38 \mathrm{G}$ & 92.32 & 43.65 & 90.26 & 7.16 & 83.1 \\
\hline CCRI36-0II & $54,872,602$ & $54,020,096$ & $8.1 \mathrm{G}$ & 92.38 & 43.63 & 89.86 & 7.08 & 82.78 \\
\hline CCRI36-0III & $54,595,684$ & $53,962,178$ & $8.09 \mathrm{G}$ & 92.66 & 43.8 & 91.23 & 7 & 84.23 \\
\hline CCRI26-1I & $52,215,302$ & $51,603,780$ & $7.74 \mathrm{G}$ & 92.55 & 43.8 & 91.65 & 7.36 & 84.29 \\
\hline CCRI36-1॥ & $49,878,906$ & $49,240,112$ & $7.39 \mathrm{G}$ & 92.31 & 43.84 & 90.75 & 7.61 & 83.15 \\
\hline CCRI36-1III & $60,433,302$ & $58,873,730$ & $8.83 G$ & 90.45 & 43.61 & 87.52 & 8.39 & 79.13 \\
\hline CCRI36-2I & $55,215,736$ & $54,486,388$ & $8.17 \mathrm{G}$ & 92.35 & 43.5 & 91.02 & 7.44 & 83.59 \\
\hline CCRI36-2II & $53,009,636$ & $52,206,706$ & $7.83 \mathrm{G}$ & 91.73 & 43.99 & 89.17 & 6.86 & 82.3 \\
\hline CCRI36-2III & $43,962,200$ & $43,194,444$ & $6.48 \mathrm{G}$ & 93.13 & 43.6 & 87.69 & 6.63 & 81.06 \\
\hline MBI8255-0| & $46,707,676$ & $45,868,652$ & $6.88 \mathrm{G}$ & 92.4 & 43.57 & 90.9 & 7.11 & 83.79 \\
\hline MBI8255-0|| & $51,016,406$ & $50,290,518$ & $7.54 \mathrm{G}$ & 92.31 & 43.68 & 90.35 & 7.25 & 83.1 \\
\hline MBI8255-0III & $48,958,146$ & $48,291,880$ & $7.24 \mathrm{G}$ & 92.27 & 43.87 & 90.48 & 7.16 & 83.32 \\
\hline MBI8255-1I & $47,850,214$ & $47,192,996$ & $7.08 \mathrm{G}$ & 92.58 & 43.44 & 90.71 & 7.31 & 83.4 \\
\hline MBI8255-1|| & $51,331,338$ & $50,606,826$ & $7.59 \mathrm{G}$ & 92.79 & 43.64 & 91.23 & 8.05 & 83.19 \\
\hline MBI8255-1III & $53,415,870$ & $52,706,364$ & $7.91 \mathrm{G}$ & 92.84 & 43.76 & 90.53 & 7.17 & 83.36 \\
\hline MBI8255-2I & $47,814,682$ & $46,903,434$ & $7.04 \mathrm{G}$ & 91.38 & 43.48 & 89.53 & 7.1 & 82.44 \\
\hline MB|8255-2|| & $42,917,798$ & $42,231,086$ & $6.33 \mathrm{G}$ & 91.92 & 43.61 & 90.29 & 7.1 & 83.19 \\
\hline MBI8255-2III & $51,881,984$ & $50,993,450$ & $7.65 G$ & 91.74 & 43.72 & 89.2 & 7.15 & 82.05 \\
\hline Average & $51,270,382$ & $50,474,857$ & $7.57 \mathrm{G}$ & 92.23 & 43.68 & 90.13 & 7.27 & 82.86 \\
\hline
\end{tabular}

result from the CSSL with distinct $G$. barbadense chromosomal segments.

\section{Identification of differentially expressed genes (DEGs)}

To investigate the differential responses to V991 infection, three replications per sample in the two lines were merged to conduct pairwise comparisons, and DEGs were identified by DESeq $R$ package (v1.18.0) and were based on the Q-value, with a false discovery rate (FDR)-adjusted cut-off value of $<0.01$ and an absolute $\log _{2}$ Change $\geq 2$. A total of 23,180 genes containing 1816 novel genes were differentially expressed in response to V991 infection (Additional file 1). These were subjected to GO enrichment analysis (Fig. 4). Forty-six subcategories were clustered into three categories, specifically biological process, cellular component, and molecular function, and the primary enriched groups in these categories were metabolic process and cellular process, cell and cell part, and binding and catalytic activity.

The results of the pairwise comparisons were as follows (Fig. 5): 1424 DEGs from CCRI36-0 vs. MBI8255-0, 8122 DEGs from CCRI36-0 vs. CCRI36-1, 167 DEGs from CCRI36-1 vs. CCRI36-2, 16,441 DEGs from MBI8255-0 vs. MBI8255-1, 4555 DEGs from MBI8255-1 vs. MBI8255-2, 8546 DEGs from MBI8255-1 vs. CCRI36-1, and 1110 DEGs from MBI8255-2 and CCRI36-2. Based on the comparisons between CCRI36-0 vs. CCRI36-1 and CCRI36-1 vs. CCRI36-2 (Fig. 6a), 53 DEGs were commonly identified, while 2644 common DEGs were found in the comparisons of MBI8255-0 vs. MBI8255-1 and MBI8255-1 vs. MBI8255-2 (Fig. 6b). There were 450 and 211 common DEGs identified from the comparisons between CCRI36-0 vs. MBI8255-0 and CCRI36-1 vs. MBI8255-1/CCRI36-2 vs. MBI8255-2 (Fig. 6c), respectively, while 508 common DEGs were observed between CCRI36-1 vs. MBI8255-1 and CCRI36-2 vs. MBI82552, ultimately identifying 115 common genes derived from all of the above comparisons.

\section{Analysis of gene temporal expression patterns}

To investigate the temporal patterns of all the 23,180 DEGs, Short Time-series Expression Miner (STEM) analyses were conducted in the two lines. In total, 19,044 DEGs in CCRI36 and 20,316 DEGs in MBI8255 were clustered into eight profiles (Fig. 7), with each profile representing a group of genes with a similar expression pattern (Additional file 2). Among the CCRI36 profiles, 11,827 DEGs $(62.1 \%$ of 19,044$)$ were further clustered into three profiles ( $P$-value $\leq 0.05)$, including one up-regulated pattern, namely, profile 7 (6024 DEGs, 31.63\%), and two down-regulated patterns, namely, profile 2 (4142 DEGs, $21.75 \%$ ) and profile 1 (1481 DEGs, 7.78\%). Similarly, 13,598 DEGs $(66.93 \%$ of the 20,316 DEGs) in MBI8255 were clustered into three profiles (P-value $\leq 0.05)$. Up-regulated profile 7 and down-regulated profile 2 contained 5912 (29.12\%) and 5288 (26.03\%) DEGs, while 


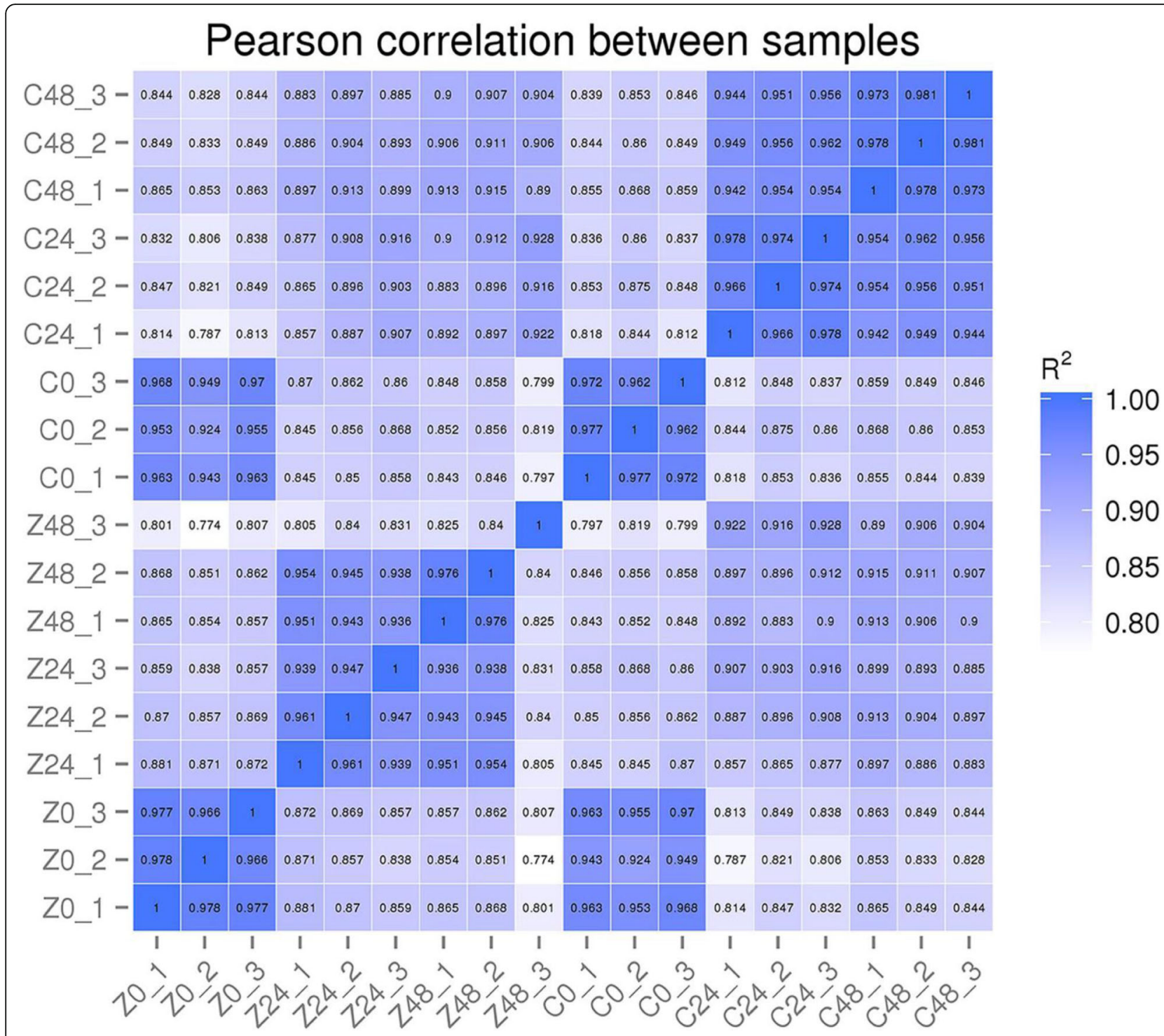

Fig. 3 Pearson correlation coefficient analysis of the total genes identified from the 18 samples. Z and C represent CCRI36 and MBI8255, respectively. 0 $=0 \mathrm{DAl}, 24=1 \mathrm{DAl}, 48=2 \mathrm{DAl}$

profile 0 (2393 DEGs, 11.78\%) first decreased and then increased in expression levels.

Next, GO enrichment analysis was conducted to identify the putative functional genes from the profiles in the two lines. Profile 7 and profile 2 possessed the most DEGs with significant values $(P$-value $\leq 0.05)$ among the eight profiles (Fig. 8). All of the DEGs were classified into three main categories consisting of biological process, molecular function, and cellular component. In combination with the GO term results of the two profiles in CCRI36 and MBI8255, metabolic process and cellular process were the dominant subcategories in biological process, while binding and catalytic activity were the most abundant subcategories in molecular function, and cell part and cell were the top two abundant subcategories in cellular component. In the up-regulated profile 7 , more DEGs clustered into the subcategories in CCRI36 than in MBI8255, while on the contrary, a greater number of DEGs clustered into the same subcategories in MBI8255 than in the down-regulated profile 2.

Similarly, the above-mentioned two profiles in CCRI36 and MBI8255 were subjected to KEGG pathway analysis, and the 20 top signaling pathways with the greatest number of DEGs are shown in Table 3. For the up-regulated pattern of profile 7 , eight common pathways were significantly enriched in the two lines, namely, phenylpropanoid biosynthesis (ko00940), plant hormone signal transduction (ko04075), circadian rhythm-plant (ko04712), flavonoid biosynthesis (ko00941), stilbenoid, diarylheptanoid and gingerol biosynthesis (ko00945), limonene and pinene 


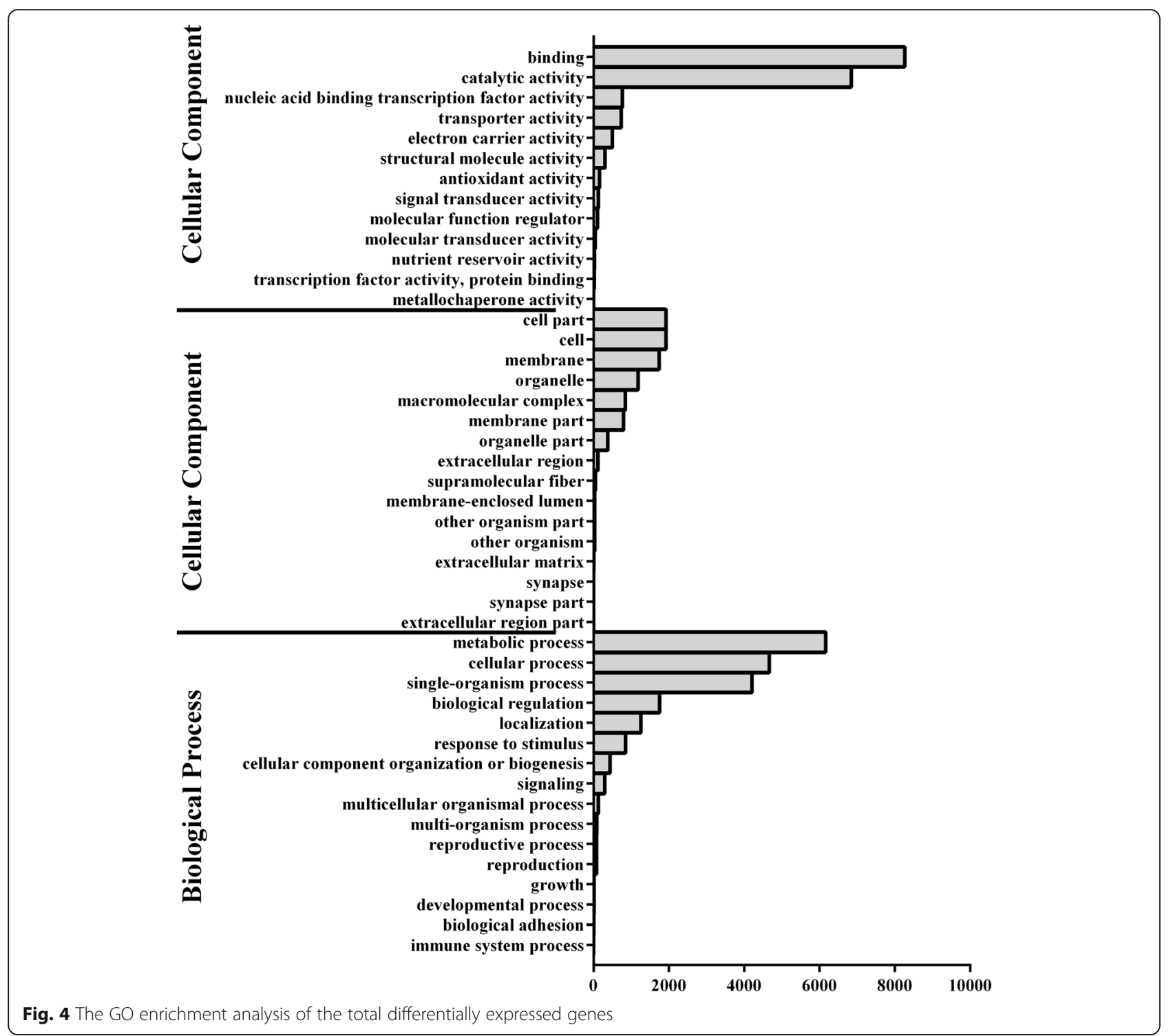

degradation (ko00903), galactose metabolism (ko00380), and carotenoid biosynthesis (ko00052). In addition to the above-mentioned pathways, there were only three significantly enriched pathways in CCRI36, including amino sugar and nucleotide sugar metabolism (ko00520), glycolysis/gluconeogenesis (ko00010), and tryptophan

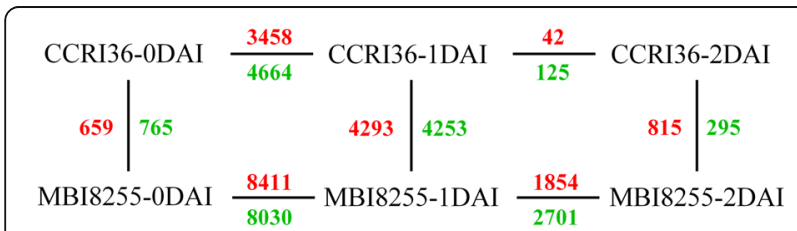

Fig. 5 The differentially expressed genes between the different samples. Red and blue numbers represent the up-regulated and down-regulated genes, respectively metabolism (ko00240). The most enriched pathway in profile 7 of the two lines was similarly annotated to starch and sucrose metabolism (ko00500), while the smallest $P$-value pathways were separately identified as flavonoid biosynthesis in CCRI36 and as phenylpropanoid biosynthesis in MBI8255.

In the down-regulated profile 2, only two common enriched pathways with significance, namely, phenylpropanoid biosynthesis and plant hormone signal transduction, were observed in the two lines, with the latter pathway indicating not only the smallest $P$-values in both CCRI36 and MBI8255, but also the greatest number of DEGs in MBI8255. Starch and sucrose metabolism was the most enriched pathway in profile 2 of CCRI36. Compared to the pathways in profile 2 of CCRI36, circadian rhythm-plant, flavonoid biosynthesis, 
A

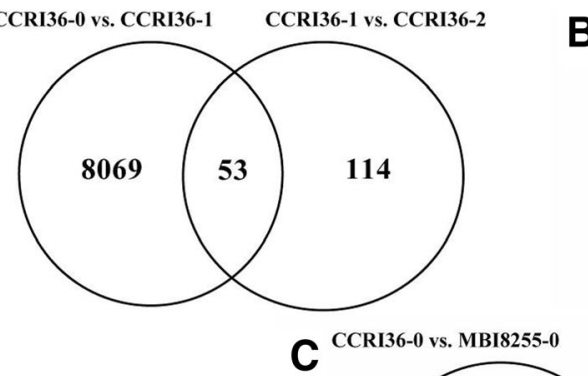

B

MBI8255-0 vs. MBI8255-1 MBI8255-1 vs. MBI8255-2

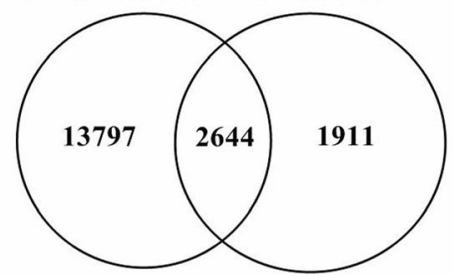

CCRI36-1 vs. MBI8255-1

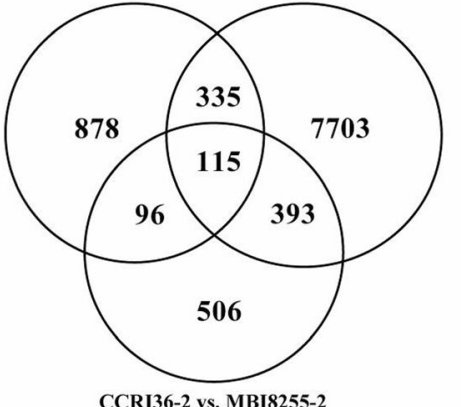

Fig. 6 The differentially expressed genes from multiple comparisons between the different samples. a represents the comparisons of CCRI36 samples at the different stages, (b) represents the comparisons of MBI8255 samples at the different stages, (c) represents the comparisons of 2 lines at the same stages

stilbenoid, diarylheptanoid and gingerol biosynthesis, and limonene and pinene degradation were the significantly enriched pathways in MBI8255 only.

\section{Expression profiling of DEGs related to the phenylpropanoid metabolic pathway}

During the process of plant growth and development, some phenylpropanoid metabolites differentially accumulate in particular cells or tissues, such as the lignins deposited in the xylem tissue and vascular bundles and

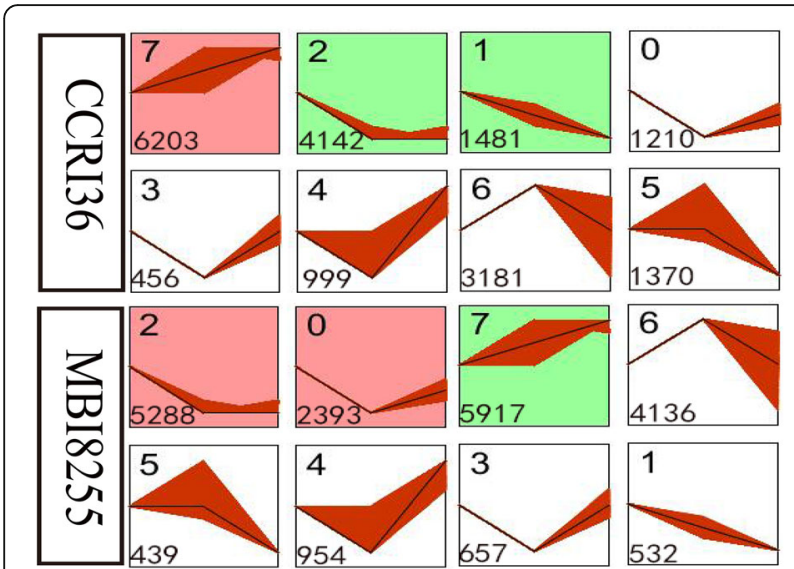

Fig. 7 Different gene expression patterns by STEM software in two lines. Each square represents a trend of gene expression. The number in top left corner indicates the profile ID number, and the number in bottom left corner indicates the number of gens in that profile. The clusters and profiles were ordered based on the number of genes and significance (default), respectively the flavonoids concentrated in the vacuolar and wall compartments, which has been reported to play important roles in plant defense $[49,50]$. The expression profiles of key enzyme genes involved in lignin and flavonoid biosynthesis were evaluated in this study (Fig. 9). The majority of up-regulated genes were related to lignin biosynthesis and the majority of down-regulated genes were related to flavonoid biosynthesis in the two lines during the preliminary stages of V991 infection (Additional file 3).

At the beginning of phenylpropanoid biosynthesis (from phenylalanine to p-coumaroyl CoA), the genes of phenylalanine ammonia-lyase (PAL), cinnamic acid.

4-hydroxylase $(\mathrm{C} 4 \mathrm{H})$, and 4-hydroxycinnamoyl-Coa ligase (4CL) exhibited almost no change from 0 DAI to 2 DAI in the two lines, except for down-regulation following the up-regulation of CCRI36 $\mathrm{C} 4 \mathrm{H}$ and the continuous up-regulation of MBI8255 4CL. In the flavonoid biosynthesis pathway, the genes of chalcone synthase (CHS) and flavanone 3-hydroxylas (F3H) were persistently up-regulated, while the chalcone isomerase (CHI) and flavonol synthase (FLS) genes showed no significant change in the two lines, except for the continuous down-regulation of the FLS gene in CCRI36. With regards to lignin biosynthesis, continuously up-regulated expression patterns in the two lines were observed in the genes of cinnamoyl-CoA:NADPH oxidoreductase (CCR), coumarate-3-hydroxylase $(\mathrm{C} 3 \mathrm{H})$, caffeoyl-CoA O-methyl-transferase (CCoAOMT), hydroxycinnamoylCoA:shikimate hydroxycinnamoyltransferase (HCT), and cinnamyl alcohol dehydrogenase (CAD), while the caffeic acid O-methyltransferase (COMT) and 


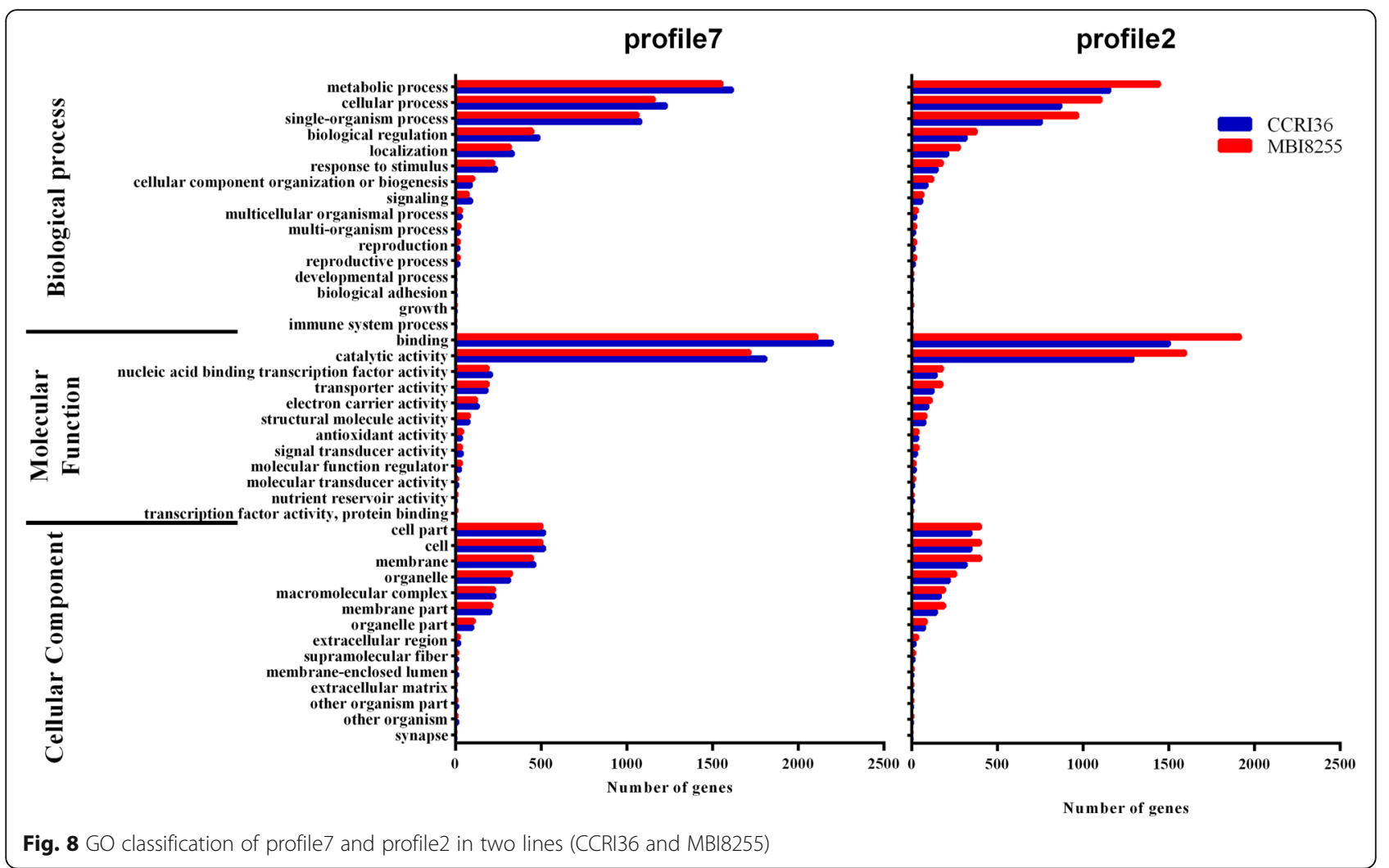

coniferaldehyde/ferulate 5-hydroxylase $(\mathrm{F} 5 \mathrm{H})$ genes were down-regulated and exhibited little change from 0 DAI to 2 DAI. In MBI8255, hydroxycinnamoyl-CoA:shikimate hydroxycinnamoyltransferase (HCT), laccase (LAC), and POD genes were first up-regulated and then down-regulated. In contrast, the former exhibited no change in CCRI36, while the latter two were up-regulated.

\section{Expression profiling of the DEGs involved in oxidation- reduction processes}

Reactive oxygen species (ROS) have been reported to play significant roles in plant growth, development, and stress defense [51] and control redox homeostasis balance between ROS-producing and ROS-scavenging pathways. Based on the GO enrichment analysis in all of the DEGs, 1433 DEGs were found to participate in the oxidation-reduction process (GO: 0055114), which served as the basis for all the downstream analyses of ROS-related genes (Fig. 10).

NADPH oxidases, also known as respiratory burst oxidase homologs (RBOHs), play significant roles in the generation of ROS in plants [52]. In the present study, a total of 11 Rboh-like protein genes were identified, namely, 2 RbohB, 5 RbohD, and 4 RbohF in the oxidation-reduction process, with the former 2 mostly showing up-regulated patterns and the latter being down-regulated in response to V991 infection in the two lines.

Simultaneously, expression pattern analysis was also conducted on the DEGs involved in ROS scavenging pathways. Sixteen ascorbate peroxidase (APX) protein genes were identified that were mostly down-regulated in the two lines. There were eight SOD genes identified from our RNA-Seq data, most of which were first down-regulated and then up-regulated in CCRI36, but down-regulated in MBI8255. However, the two SOD genes annotated as copper/zine SOD 1 and manganese SOD 1 presented continuous up-regulation in CCRI36 and down-regulation following up-regulation in MBI8255, which was consistent with the aforementioned biochemical investigations of root SOD. Five catalase (CAT) genes were mostly down-regulated in CCRI36 and first down-regulated and then up-regulated in MBI8255, which corroborated the aforementioned CAT investigations. One glutaredoxin (GRX) gene exhibited no significant change in CCRI36, but presented a down-regulated pattern in MBI8255. In addition, the genes of eight monodehydroascorbate reductase (MDAR), seven glutathione peroxidase (GPX), and three peroxiredoxin (PrxR) genes were mainly down-regulated in CCRI36, but showed up-regulation following down-regulation in MBI8255. In both lines, one ferritin and three thioredoxin (Trx) genes presented up-regulated expression patterns, while most of 
Table 320 top KEGG pathways with high representation of the DEGs

\begin{tabular}{|c|c|c|c|c|c|}
\hline \multirow[t]{2}{*}{ Pathway } & \multicolumn{4}{|c|}{ DEGs with pathway annotation } & \multirow{2}{*}{$\begin{array}{l}\text { Pathway } \\
\text { ID }\end{array}$} \\
\hline & $\begin{array}{l}\text { CCRI36 } \\
\text { profile7 }\end{array}$ & $\begin{array}{l}\text { MBI8255 } \\
\text { profile7 }\end{array}$ & $\begin{array}{l}\text { CCRI36 } \\
\text { profile2 }\end{array}$ & $\begin{array}{l}\text { MBI8255 } \\
\text { profile2 }\end{array}$ & \\
\hline Starch and sucrose metabolism & 76 & 72 & 50 & 57 & ko00500 \\
\hline Phenylpropanoid biosynthesis & $73^{*}$ & $71^{*}$ & $49^{*}$ & $56^{*}$ & ko00940 \\
\hline Plant hormone signal transduction & $60^{*}$ & $55^{*}$ & $45^{*}$ & $58^{*}$ & ko04075 \\
\hline Circadian rhythm - plant & $59^{*}$ & $56^{*}$ & 36 & $48^{*}$ & ko04712 \\
\hline Flavonoid biosynthesis & $52^{*}$ & $42^{*}$ & 25 & $33^{*}$ & ko00941 \\
\hline Carbon metabolism & 49 & 38 & 25 & 37 & ko01200 \\
\hline Stilbenoid, diarylheptanoid and gingerol biosynthesis & $47^{*}$ & $46^{*}$ & 26 & $40^{*}$ & ko00945 \\
\hline Limonene and pinene degradation & $45^{*}$ & $46^{*}$ & 27 & $39^{*}$ & ko00903 \\
\hline Ribosome & 45 & 46 & 32 & 43 & ko03010 \\
\hline Amino sugar and nucleotide sugar metabolism & $43^{*}$ & 38 & 20 & 28 & ko00520 \\
\hline Pentose and glucuronate interconversions & 36 & 31 & 26 & 27 & ko00040 \\
\hline Biosynthesis of amino acids & 32 & 35 & 24 & 31 & ko01230 \\
\hline Glycolysis/Gluconeogenesis & $28^{*}$ & 23 & 9 & 9 & ko00010 \\
\hline Protein processing in endoplasmic reticulum & 28 & 20 & 13 & 22 & ko04141 \\
\hline Pyrimidine metabolism & 28 & 27 & 24 & 25 & ko00240 \\
\hline Tryptophan metabolism & $26^{*}$ & 14 & 12 & 11 & ko00380 \\
\hline Galactose metabolism & $24^{*}$ & $28^{*}$ & 6 & 10 & ko00052 \\
\hline Carotenoid biosynthesis & $23^{*}$ & $24^{*}$ & 13 & 17 & ko00906 \\
\hline Plant-pathogen interaction & 23 & 16 & 13 & 19 & ko04626 \\
\hline Ubiquitin mediated proteolysis & 23 & 16 & 13 & 18 & ko04120 \\
\hline
\end{tabular}

"*" means the significantly enriched pathways

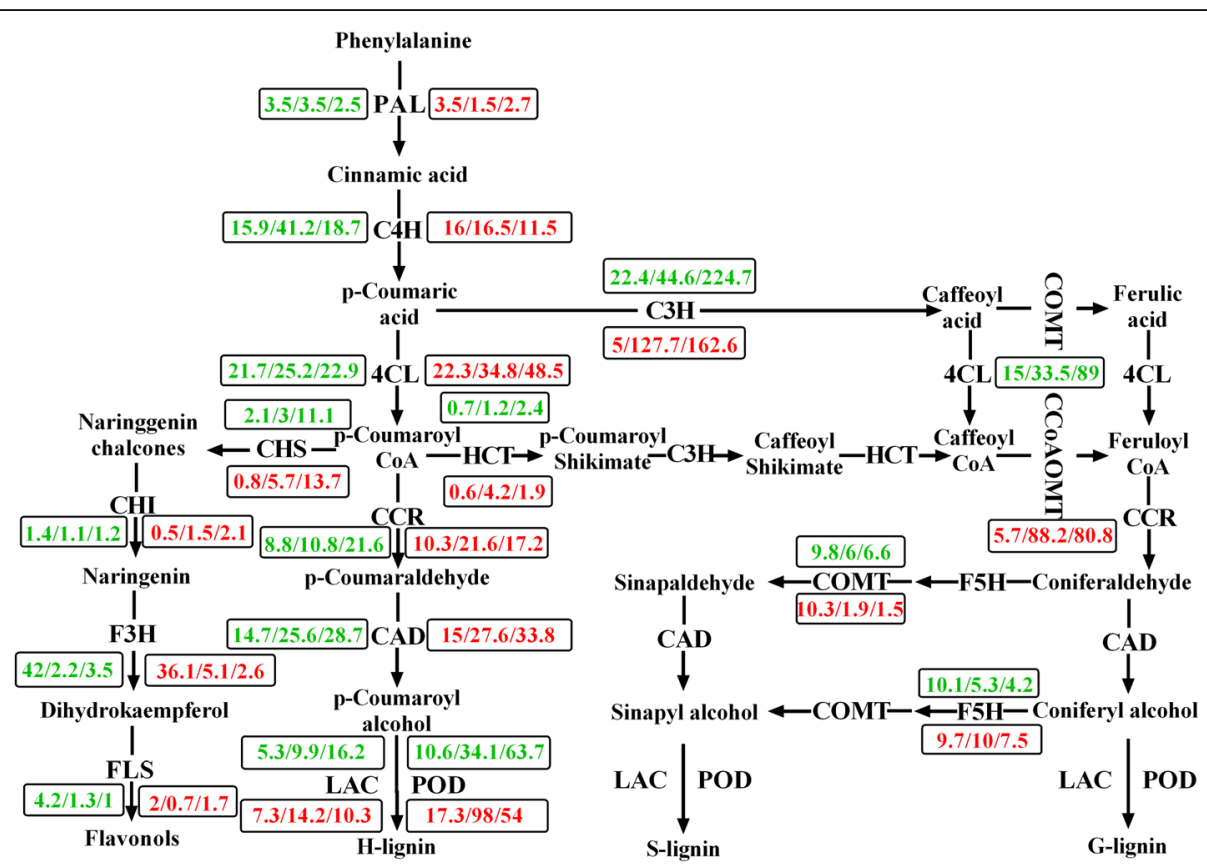

Fig. 9 The expression of DEGs related with phenylpropanoid biosynthesis pathway. Red and blue numbers represent the DEGs from MBI8255 and CCRI36, respectively 


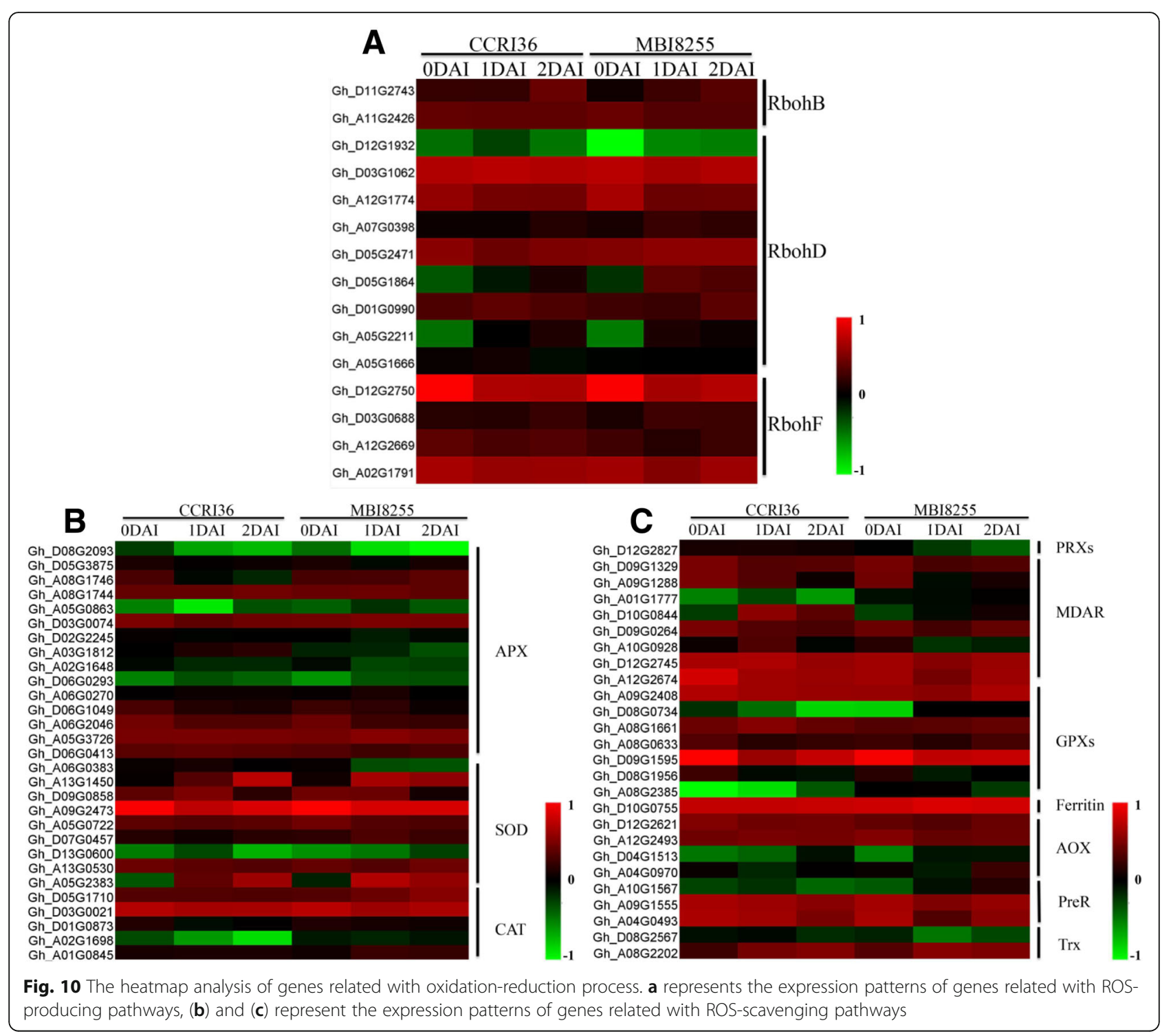

the four alternative oxidase (AOX) genes were first down-regulated and then up-regulated. The correlation of all of the complicated and diverse changes in the above-mentioned genes with ROS-producing and ROS-scavenging pathways indicated that the oxidationreduction process plays extraordinary roles in maintaining the redox homeostasis balance under V991 infection.

\section{Expression profiling of DEGs relevant to plant resistance} Based on the GO enrichment analysis on all the DEGs, a total of 182 DEGs were enriched in the biological process of defense response (GO:0006952) and were further analyzed in combination with the functional annotation of $A$. thaliana, eventually obtaining 158 immunity-related DEGs (Fig. 11). In the present study, most DEGs associated with plant resistance to VW were annotated as $\mathrm{R}$ genes, which were classified into CC-NBS-LRR and TIR-NBS-LRR genes. Eleven CC-NBS-LRR protein genes were identified, of which Gh_D05G0053 and Gh D05G3603 with relatively high levels gradually increased in the two lines (except for Gh_D05G0053 from 1 DAI to 2 DAI). There were 25 genes encoding TIR-NBS-LRR proteins, and four highly expressed genes were observed, namely, Gh_A10G2076, Gh_A09G2289, Gh_D03G1355, and Gh_A11G2680. The first and middle two genes were separately up-regulated and down-regulated after being first up-regulated in both lines, while the last gene showed continuous down-regulation in MBI8255 and up-regulation following down-regulation in CCRI36. In addition to the above-mentioned NBS-LRR proteins, we also found 22 genes encoding LRR and NB-ARC domain-containing disease resistance proteins and 42 genes encoding an NB-ARC domain-containing disease resistance protein in 

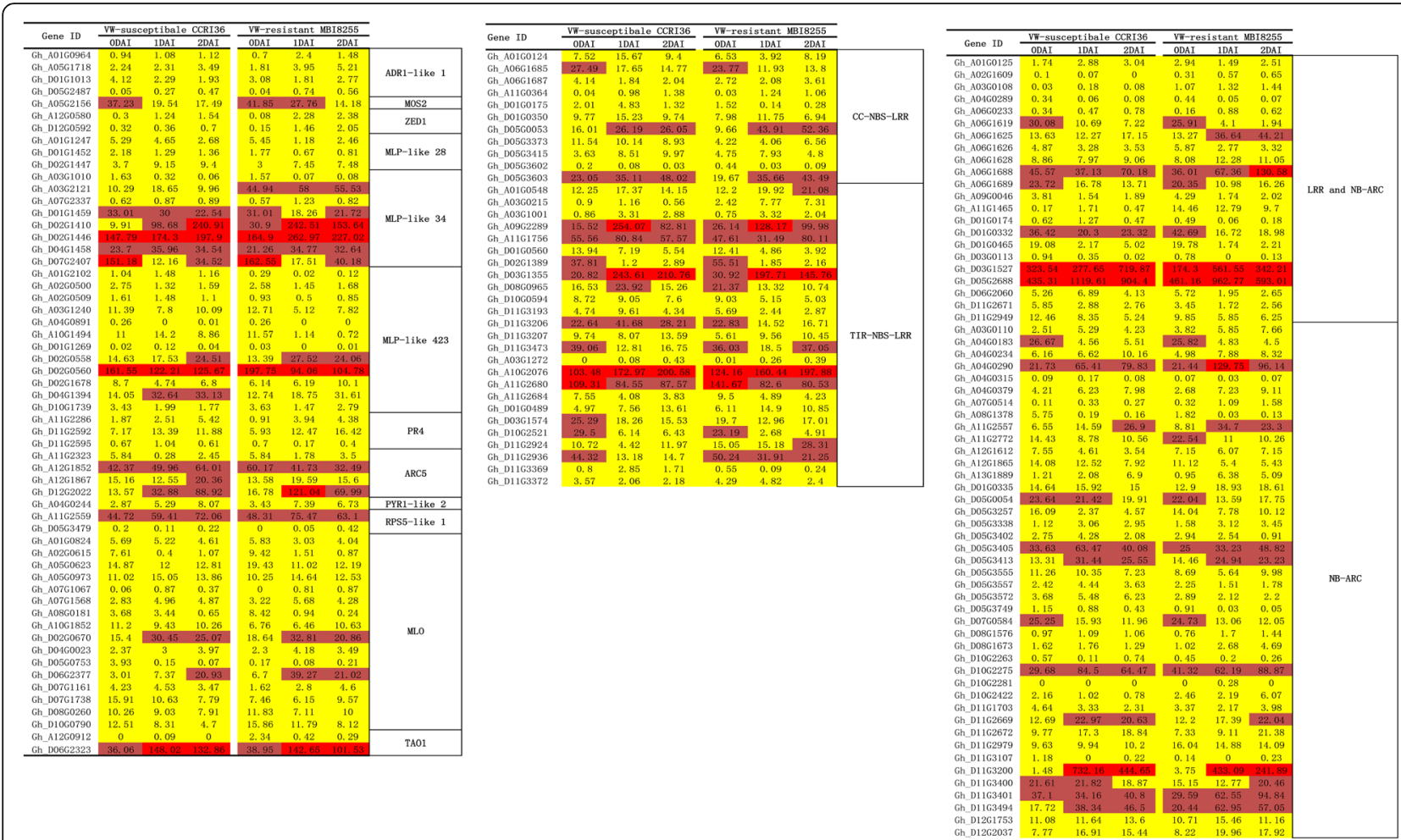

Fig. 11 The expression pattern analysis of genes related with Defense response

this GO term. Among the LRR and NB-ARC genes, Gh_D05G2688 and Gh_D03G1527 exhibited extremely high expression levels, with the former being first up-regulated and then down-regulated in the two lines, while the latter was up-regulated following down-regulation in CCRI36 and down-regulation following up-regulation in MBI8255. As for the NB-ARC genes, Gh_D11G3200 exhibited the highest expression and was first up-regulated and then down-regulated in the two lines.

Many other genes participate in plant resistance to VW, the expression patterns of which were further analyzed as follows. Activated disease resistance 1 (ADR1) genes, including ADR1, ADR1-like1, and ADR1-like2, were correlated with the regulation of the basal and ETI mediated defense in Arabidopsis [53]. Four ADR1-like1 genes were identified, namely, Gh_A01G0964, Gh_A05G1718, Gh_ D05G2487, and Gh_D01G1013, in which the transcription patterns were mostly up-regulated in CCRI36 and down-regulated following up-regulation in MBI8255.

MOS2, also described as a D111/G-patch domain-containing protein, is essential for innate immunity in $A$. thaliana [54]. One MOS2 gene, Gh_A05G2156, was continuously down-regulated in the two lines, the expression level of which in MBI8255 was higher than in CCRI36, not only at 0 DAI but also at 1 DAI.
ZED1, also known as HOPZ-ACTIVARED RESISTANCE 1, is responsible for high temperature-dependent autoimmunity and growth retardation in zed1-D [55]. Two ZED1 genes, identified as Gh_A12G0580 and Gh_D12G0592, presented a gradually increasing expression level from 0 DAI to 2 DAI in the two lines.

Major latex protein (MLP)-like proteins belong to the Bet $\mathrm{v} 1$ family, also described as the pathogenesis related 10 (RP 10)-like protein family, the expression patterns of which are related to defense or stress responses [56]. Gh_A01G1247, Gh_D01G1452, and Gh_D02G1447 were annotated as MLP-like protein 28 genes, of which the former two were up-regulated following down-regulation (except for down-regulation in Gh_A01G1247), whereas the latter was up-regulated from 0 DAI to 2 DAI in the two lines. Eight MLP-like 34 were identified, and the expression levels of Gh_D02G1410, Gh_D02G1446, and Gh_D07G2407 were relatively high in comparison to the remainder of the genes, with the former two being up-regulated and the latter being first down-regulated and then up-regulated in response to V991 infection in CCRI36 and MBI8255. There were 12 MLP-like 423 genes, and Gh_D02G0560 had extremely high RPKM values relative to the remainder, indicating up-regulation following down-regulation in both lines. 
Three genes related to Arabidopsis pathogenesis-related 4 [57] were found, and the transcript level of Gh_D11G2592 was relatively high compared to Gh_A11G2286 and Gh_D11G2595, which were first up-regulated and subsequently down-regulated in CCRI36. In contrast, these were continuously up-regulated in MBI8255, obtaining higher expression levels in the VW-resistant line than the VW-susceptible line at 2 DAI.

A resistance $(\mathrm{R})$ gene in rice belonging to $\mathrm{P}$-loop containing the nucleoside triphosphate hydrolases superfamily protein has been implicated in the signaling pathways of the rice blast (Magnaporthe grisea) resistance reaction [58]. Four P-loops containing nucleoside triphosphate hydrolases superfamily protein genes, including Gh_A11G2323, Gh_A12G1867, Gh_A12G1852, and Gh_D12G2022, were identified, and the former two presented up-regulation after down-regulation, while the latter two were gradually up-regulated in CCRI36. In contrast, the first gene was up-regulated following down-regulation, the second and forth were down-regulated after being up-regulated, while the third was continuously down-regulated in MBI8255.

Pyrabactin resistance (PYR)/PYR1-like (PYL)/regulatory components of ABA receptors (RCAR) belong to the $\mathrm{ABA}$ receptor family, and $\mathrm{ABA}$ plays significant roles in plant abiotic stress resistance [59]. One PYL2 gene, Gh_A04G0244, was up-regulated in CCRI36, but was down-regulated after being up-regulated in MBI8255.

Resistance to Pseudomonas syringae 5 (RPS5), encoding the coiled-coil nucleotide-binding site leucine-rich repeat (CC-NBS-LRR) domain, recognizes the Avirulence effector protein Pseudomonas phaseolocola B (AvrPphB) from Pseudomonas syringae [60, 61]. Two RPS5-like genes were found in the results, namely, Gh_A11G2559 and Gh_D05G3479, of which the former with higher FPKM values than the latter was up-regulated in CCRI36, but down-regulated following up-regulation in MBI8255.

The mildew locus $\mathrm{O}$ (MLO) gene family, ubiquitously found in most land plants, encodes seven transmembrane (TM) domain proteins that participate in the regulation of defense and cell death in response to biotic and abiotic stress [62]. Fifteen genes annotated as MLO family proteins demonstrated diverse expressional abundances. Gh_D02G0670 and Gh_D06G2377 had relatively high RPKM values and were first up-regulated and then down-regulated in the two lines, except for the latter, which was consistently up-regulated in CCRI36. Interestingly, the expression levels in MBI8255 were higher than in CCRI36, except for Gh_D02G0670 at 2 DAI.

The target of AvrB operation 1 (TAO1), belonging to the TIR-NB-LRR protein family, was reported to contribute to disease resistance in response to the
Pseudomonas syringae effector AvrB in A. thaliana [63]. Two TAO1 genes were identified, and Gh_D06G2323 was first up-regulated and then down-regulated in the two lines and exhibited significantly higher expression levels than Gh_A12G0912.

\section{Validation of the RNA-Seq results by quantitative real- time (qRT)-PCR}

To confirm the reliability of our RNA-Seq results, 20 DEGs were randomly selected for qRT-PCR (Fig. 12). The primer sets are listed in Table 4. The reference gene was the house-keeping $\beta$-Actin gene, and the expression patterns of the 20 genes in the qRT-PCR results were highly consistent with the transcriptome sequencing data, which further supported the reliability of our RNA-Seq data.

\section{Discussion}

\section{Experimental conditions and phenotypic evaluation}

In the present study, VW resistance evaluations were conducted on the CSSL, its recurrent parent, and the two controls under the artificial greenhouse, natural field, and disease nursery tests. The disease index can be calculated based on the number of leaves with symptoms of necrosis and chlorosis. Although three replications in the greenhouse tests were carried out with different numbers of paper cups (five seedlings per cup; the first replication included five cups, the second replication included seven cups, and the third replication contained 10 cups), the total number of seedlings for either the resistant control (Zhongzhimian2) or the susceptible control (Jimian11) was sufficient for the disease evaluation. This not only increases the accuracy, but also reduces the experimental error. Based on the greenhouse results, the DI values of MBI8255 and CCRI36 were relatively close to those of Zhongzhimian2 and Jimian11 at 15 DAI, respectively. A similar phenomenon was observed at $30 \mathrm{DAI}$, even though higher DI values were observed than at 15 DAI, which indicated that the selected CSSL and its recurrent parent were separately resistant and susceptible to V991.

In the natural field tests, the VW resistance evaluations were performed on the four lines as in the greenhouse investigations in two different locations (one replication in Anyang and one replication in Xinjiang), and were investigated at the flowering and maturation stages. The two resistant lines (MBI8255 and Zhongzhimian2) and two susceptible lines (CCRI36 and Jimian11) in Anyang presented relatively close DI values at both the flowering and mature stages. A similar trend was observed in Xinjiang, whereby MBI8255 exhibited VW resistance, while CCRI36 demonstrated VW susceptibility in the natural environment. The four lines in Anyang showed higher DI values than those in Xinjiang at the 


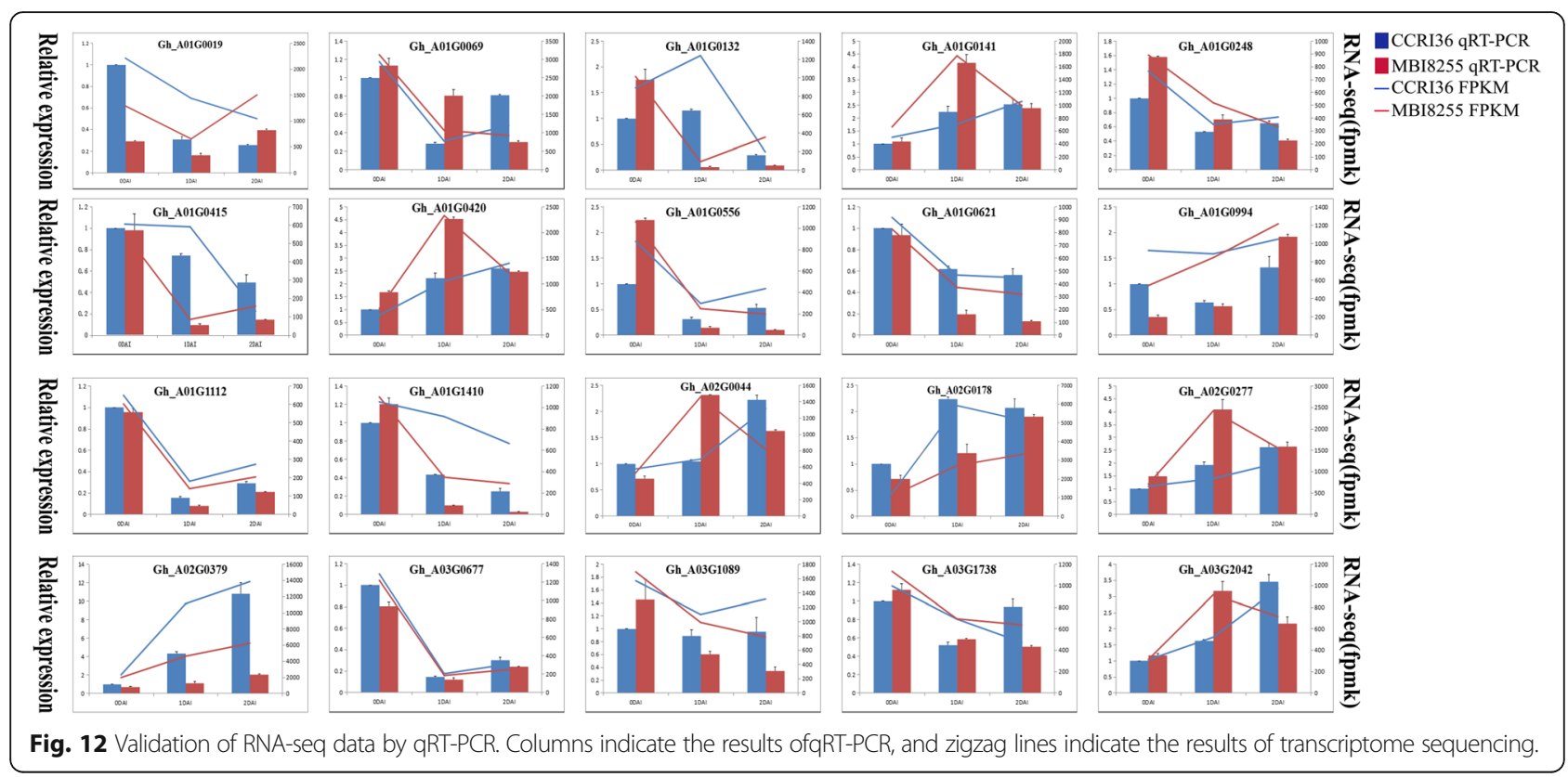

flowering stage, but presented relatively low DI values in Anyang at the mature stage, except in CCRI36. These variations in the field results could be attributed to a variety of factors, such as the infection of plants by a combination of VW strains, different locations with diverse quantities and virulence levels of soil fungus, and various developmental and environmental conditions [64]. For the disease nursery tests, only one replicate of MBI8255, CCRI36, and Jimian11 was conducted in Kaifeng, while the susceptible control showed $56.77 \%$ DI,

Table 4 Primers used for qRT-PCR validation

\begin{tabular}{|c|c|c|}
\hline Gene ID & Forward $5^{\prime}-3^{\prime}$ & Reverse $5^{\prime}-3^{\prime}$ \\
\hline Gh_A01G0019 & CGTCATCGGCCACAAGAAAC & CATGTAAGCCACCGCCCTTA \\
\hline Gh_A01G0069 & TTGGGTGGATGAAACCCGAG & GACGGACTTCCTCCGATTC \\
\hline Gh_A01G0132 & GCCAATAACAAACTCGGCGG & CACCCGAAGGAACAATCCCA \\
\hline Gh_A01G0141 & GGTITTCAAGGGTCTGGGCT & CGCTACTTTCTCCCTTGGCT \\
\hline Gh_A01G0248 & ATGCGGAGGAGGCTTGTAG & TCTTTCGTTCCGGCTTGGT \\
\hline Gh_A01G0415 & CAGGGCAAGAAAAGCAGTCC & GTTCAGACCCACCACACCA \\
\hline Gh_A01G0420 & CCGATGGTTGGTTGCTGAAC & GAAGTGGGAGTCAAGAGCC \\
\hline Gh_A01G0556 & CTGGTGGAACTTCTTACACGC & CCTGCTGTCTGTGGTCTAC \\
\hline Gh_A01G0621 & CAGCATTTGAGTGGACAGC & ATGAACACCGACAAGCCGAA \\
\hline Gh_A01G0994 & CGCCACTCTACGATCCTTCC & CCAAACCTTGCGCCGTTAAA \\
\hline Gh_A01G1112 & CCCAAGTTAGCATGTTGAAGGC & GGGAAACGATCAAAGAGAAGCC \\
\hline Gh_A01G1410 & TGACTCTCTTCGGCGTCAAC & GCCATTTTGCCACCACTGAG \\
\hline Gh_A02G0044 & TGGTACGGAGACTGTGTGGTA & TCACGTAATCCCAGATCCTCG \\
\hline Gh_A02G0178 & CTCGTTGCACAAAGTGGTGG & TITACGCCCTGGTTCTCCC \\
\hline Gh_A02G0277 & GGGTTCTATGTGGTGGCTC & CCACAACCTTAGCACCCCAA \\
\hline Gh_A02G0379 & TTATCAGCCTCCGAAGAAGCC & TGGAGGGTGTCCTGGATAGT \\
\hline Gh_A03G0677 & AACCTCCTTCCATTCCACGC & TGCTTATGCCCCTTCCGATG \\
\hline Gh_A03G1089 & GGCGACTTGCAGAAAGAAAGG & CAGTTITTCCCTCCGCTGG \\
\hline Gh_A03G1738 & CAACCGAGGAACGCAGGATA & CTCCCCTTCCGCTTGCTTTA \\
\hline Gh_A03G2042 & GCTTCCGGTCAAGTGGAGAA & CTCACGCGCTTCATCCCTAT \\
\hline Gh_A01G0019 & CGTCATCGGCCACAAGAAAC & CATGTAAGCCACCGCCCTTA \\
\hline
\end{tabular}


which was slightly higher than in either the greenhouse or field tests. Although presenting a more similar DI to that at 15 DAI or the flowering stage of the greenhouse and field tests, MBI8255 and CCRI36 separately exhibited resistance and susceptibility to VW, which might be due to the insufficient number of sampled plants. In combination with the greenhouse, field, and disease nursery results, the selected CSSL, MBI8255, proved to be resistant to VW despite showing a slightly higher DI than the resistant control, Zhongzhimian2. Conversely, the recurrent parent, CCRI36, was susceptible to VW despite demonstrating a slightly lower DI than the susceptible control, Jimian11, which provided a solid foundation for further biochemical tests and transcriptome sequencing.

\section{Biochemical mechanisms of resistance to V991 infection}

As a result of long-term interactions with diverse pathogens, plants have evolved many strategies to prevent damage or invasion by microbes, including plant physical defense responses and physiological and biochemical resistance, of which the regulation of antioxidant enzymes and the synthesis of biochemical substances is of great significance. ROS have been shown to mediate signal transduction and regulate homeostasis as key secondary messengers, high concentrations of which could result in membrane damage and the destruction of cellular organelles and biomolecules, ultimately causing cell death [65]. In order to repair the damage caused by ROS in response to pathogen infection, plants enhance the activities of antioxidative enzymes, such as CAT, SOD, POD, and PPO. In addition to the above-mentioned enzymes, PAL is also an essential enzyme that is correlated with the synthesis of lignin and suberin and plays a key role in the regulation of the synthesis of salicylic acid (SA) and the establishment of systemic acquired resistance (SAR) [66]. With regards to the VW caused by $V$. dahliae, the POD, PAL, and PPO activities were positively correlated with VW resistance in eggplant [67], and the former two were strengthened after inoculation with the V991 strain in cotton [43]. To evaluate the relationships between key enzymes and VW resistance, three protective enzymes (CAT, SOD, and POD) and two defense enzymes (PAL and PPO) were selected and tested on root samples collected at 0,1 , and 2 DAI in MBI8255 and CCRI36. According to the activity results of the protective enzymes (Fig. 2), the activities of the VW-resistant and VW-susceptible lines at the same stage showed no significant difference either in root CAT or root SOD, which indicated that no correlation between VW resistance and CAT activity or SOD activity was detected in the present study. However, significant differences were observed in POD activity between MBI8255 and CRI36 at the same stage, except at 1 DAI, which implied a close relationship between POD activity and VW resistance. Furthermore, POD activity in MBI8255 was significantly higher than in CCRI36 at 0 and 2 DAI, which indicated that in comparison to the susceptible recurrent parent, the resistant CSSL possessed higher POD activity regardless of V991 infection. With regards to the two defense enzymes, only PPO activity in the two lines at 2 DAI differed significantly, while the VW-resistant line presented higher PAL and POD activities than the VW-susceptible line, except for PAL activity at 1 DAI. This indicated a clear correlation between VW resistance and POD activity, and furthermore, that the resistant line had higher PAL and POD activity in response to V991 infection. Based on the above results, the POD and PPO activities were positively correlated with VW resistance, which was consistent with the findings of a previous study [43, 67]. However, sufficient evidence is lacking to prove a close correlation between PAL activity and VW resistance, although higher activities were identified in the resistant line, which did not corroborate previous studies. One reason might be related to the complicated background of the selected CSSL, which should be addressed in further research.

Some biochemical substances in plants provide energy for the self-defense response or are the products of damage and injury caused by pathogen invasion, including MDA [68, 69], proline [70, 71], and soluble sugar [72], which are correlated with plant resistance. As the final product of lipid peroxidation, the root MDA contents in the two lines showed significant differences at the same stage and a down-regulated pattern, except at 0 DAI, and presented higher contents in MBI8255, which might indicate a negative correlation with VW resistance. However, an obvious correlation between VW resistance and the contents of proline, soluble sugar, and soluble protein was not detected, and no significant differences were found between either the two lines at the same stage, or between MBI8255 or CCRI36 at the different stages. Our results corroborated previous studies [43, 67], thereby providing useful information for the further analysis of the combination with our RNA-Seq data.

\section{Gene expression variation in response to V991 infection}

RNA-Seq, as a powerful tool focused on global gene expression at the transcriptome level, can reveal the mechanisms of particular biological processes and was utilized to identify the key candidate genes or significant signaling pathways in response to VW in the present study. Eighteen root samples derived from the VW-resistant MBI8255 and VW-susceptible CCRI36 were collected at 0,1 , and 2 DAI of V991 infection. Library construction and transcriptome sequencing of these samples produced a total of 908.547 million clean 
reads (approximately $136.27 \mathrm{~Gb}$ data) with more than 91.38\% of the Q30 value and over $43.44 \%$ of the GC content. No less than $87.69 \%$ of the clean reads were successfully mapped to the reference genome and were subsequently subjected to assembly, ultimately obtaining 77,412 genes, including 6934 novel genes. PCC analyses were used to confirm the relationships of the different samples, and high similarities were found among the three replicates of each sample and also in the samples from the same stages, which confirmed the reliability of our RNA-Seq data.

Through multiple comparisons between the samples, 23,180 genes (1816 novel genes) were differentially expressed in response to V991 infection, with more down-regulated genes than up-regulated genes observed. Adopting STEM analysis to investigate the temporal expression patterns of the total DEGs, three profiles $(P \leq$ $0.05)$ per line were identified, and GO and KEGG enrichment analysis were performed on up-regulated profile 7 and down-regulated profile 2, which exhibited the same patterns. In the two profiles, metabolic process and cellular process, binding and catalytic activity, and cell part and cell were the most abundant subcategories in biological process, molecular function, and cellular component, respectively. The genes clustered into the same GO terms in MBI8255 and CCRI36 showed opposite patterns, and there were more enriched genes of CCRI36 over those of MBI8255 in profile 7 while presenting less enriched genes of CCRI36 than those of MBI8255 in profile 2. Based on the KEGG results, only two pathways, namely, phenylpropanoid biosynthesis and plant hormone signal transduction, in the two profiles or in the two lines, were significantly enriched, and more genes were clustered in CCRI36 than in MBI8255 in the up-regulated expression pattern (profile 7), while more genes were down-regulated in MBI8255 than in CCRI36 (profile 2). The consistency between the GO and KEGG results might be due to the diverse strategies adopted in response to V991 infection by the different resistant or susceptible lines. With regards to the enriched metabolic processes in our results, secondary metabolism pathways were found to play particularly significant roles in response to V991 infection and they have been reported to participate in the abiotic stress response [73]. Furthermore, phenylpropanoid and flavonoid biosynthesis pathways have also been suggested to accumulate in response to pathogen infection, as indicated in a previous study [74].

\section{The phenylpropanoid metabolic pathway in response to V991 infection}

The phenylpropanoid pathway in plants has been reported to participate in some abiotic stress responses, such as drought, salinity, and pathogen infection, and it serves as the main metabolic pathway for lignin biosynthesis [75]. As a major cell wall component, lignin acts as a main plant mechanical support structure and defense system in response to pathogen infection [76], the synthesis of which is subjected to the oxidative coupling of $p$-hydroxyphenyl $(\mathrm{H})$, guaiacyl $(\mathrm{G})$, and syringyl (S) monolignols. In this study, the majority of the key enzymes in the phenylpropanoid pathway were analyzed based on the combined RNA-Seq and qRT-PCR results. Diverse expression patterns were observed in response to V991 infection, mostly presented by the up-regulation of lignin biosynthesis-related enzymes rather than flavonoid synthesis-related enzymes, which might result from the antagonistic relationships between the two synthesis pathways. Nevertheless, our findings still indicate the positive effect of V991 infection on the phenylpropanoid pathway in CCRI36 and MBI8255, further promoting lignin biosynthesis.

During the synthesis pathways of $\mathrm{G}$ and $\mathrm{S}$ type lignins, most genes showed up-regulated expression patterns, including $\mathrm{C} 3 \mathrm{H}, \mathrm{CCR}$, and $\mathrm{CAD}$, while only one down-regulated gene, COMT, was identified, which represented the difficult shift from caffeic acid to ferulic acid during the synthesis of $\mathrm{S}$ and $\mathrm{G}$ type lignins. Furthermore, the up-regulated CCoAOMT, which transforms caffeoyl CoA to feruloyl CoA, might be efficiently utilized to obtain adequate lignin, as has been reported in switchgrass and duckweed [77, 78]. In general, the lignin biosynthesis pathway stimulated by V991 infection would produce a higher lignin content, which could combine with some other antioxidants to participate in the regulation of ROS production in the apoplast [76]. These results revealed the significant role of the phenylpropanoid metabolic pathway in response to V991 infection.

\section{Oxidation-reduction processes in response to V991 infection}

In response to abiotic stress, ROS generation in plants can be activated by ROS-producing genes, resulting in oxidative stress and accordingly giving rise to ROS-scavenging pathways in order to maintain the ROS homeostatic balance. In the present study, a total of 1433 DEGs were found to participate in oxidation-reduction processes, derived from the GO analysis results of all the DEGs. RBOHs, identified as RbohB, RbohD, and RbohF in our study, have been reported to participate in the process of ROS production [52] and mostly showed up-regulated patterns from 0 DAI to 2 DAI, which indicated increased oxidative stress in response to V991 infection.

In order to eliminate the generated ROS, plants have evolved ROS scavenging pathways in response to damage caused to the cellular components by high oxidative stress. Among the antioxidant enzymes, SOD, as the first 
line of antioxidant defense, is responsible for transforming $\mathrm{O}_{2}{ }^{-}$into $\mathrm{H}_{2} \mathrm{O}_{2}$, and subsequently APX and CAT further transform $\mathrm{H}_{2} \mathrm{O}_{2}$ into $\mathrm{H}_{2} \mathrm{O}$ with their corresponding compounds [79]. Under V991 infection, only two SOD genes showed up-regulated expression patterns in CCRI36, but presented down-regulation after up-regulation in MBI8255, which was consistent with the SOD results of the biochemical tests, indicating the significant roles of the two enzymes in ROS scavenging pathways. APX or CAT were mainly down-regulated in CCRI36, but were up-regulated following the down-regulation in MBI8255. Similar results were observed with regards to the activity changes of MDAR, GPXs, and PreR. In the two lines, in addition to the up-regulation and then down-regulation of AOX enzymes, ferritin and Trx were continuously up-regulated, which suggested their positive participation in ROS scavenging pathways. Therefore, under $V$. dahliae infection, ROS generation increased in both CCRI36 and MBI8255, subsequently leading to the activation of ROS scavenging pathways to protect the plants. In contrast, the resistant line exhibited relatively higher ROS scavenging-related enzyme activity than the susceptible line, which might be due to the resistant line possessing a relatively well-developed ROS scavenging system.

\section{Plant resistance in response to V991 infection}

The process of pathogen infection starts from an influx of calcium and an oxidative burst triggered by PAMPs, and subsequently activates not only the MAPK pathway and calcium-dependent protein kinase, but also stomatal closure and transcriptional reprogramming, ultimately resulting in SA accumulation and callose deposition [79-81]. In order to respond to the invasions from various pathogens, plants have evolved two main defense mechanisms. Along with the recognition of PAMPs occurring on the extracellular surface of the host cell, PPRs together with their stimulation activate a basal immune response, namely, PTI $[27,28]$. Upon successful pathogen invasion, the generated effectors are first delivered into the host cells, further suppressing the PTI, which can be recognized by specific $\mathrm{R}$ genes. ETI, the second-type immune reaction, is activated by the production of $\mathrm{R}$ genes. The induction of plant resistance to pathogens and HR are ultimately triggered following the special recognition of both PRRs and $R$ genes [30, 31].

In the present study, the interactions of two lines, MBI8255, resistant to VW, and CCRI36, susceptible to VW, with $V$. dahliae were investigated with the aim of revealing the molecular mechanisms of the plant immune response to plant pathogens. Based on the results of GO enrichment analysis of all of the DEGs, a total of 158 immunity-related genes with A. thaliana annotations were clustered into the defense response GO term
(GO: 0006952), and significantly different expression patterns were observed between the resistant and susceptible lines. Among the resistance genes, $R$ genes were mostly identified from the results and could be classified into TIR-NBS-LRR and CC-NBS-LRR genes based on the different $\mathrm{N}$-terminal domains [35, 36]. As numerous genes were annotated as $\mathrm{R}$ genes, only those with high-level expression were further investigated, although more genes encoding the second-type $\mathrm{R}$ domain were observed. With regards to the first-type $R$ genes, up-regulated expression patterns were identified in the two highly expressed genes, Gh_D05G0053 and Gh_D05G3603, with the former showing higher expression in MBI8255 than in CCRI36, except at 0 DAI, while the latter presented no significant difference between the expression levels of the two lines at the same stage. There were more than twice as many second-type $R$ genes than first-type $\mathrm{R}$ genes, and five high-expression genes were found, namely, Gh_D05G3603, Gh_A10G2076, Gh A09G2289, Gh_D03G1355, and Gh_A11G2680. The first four of these genes showed up-regulation from 0 DAI to 1 DAI, while the latter one was continuously down-regulated during the preliminary stages of V991 infection, and, interestingly, was highly expressed at 0 DAI in the resistant line. In addition, there were 22 genes encoding LRR and NB-ARC domain-containing disease resistance proteins in this term, and the two high-level expressed genes, Gh_D03G1527 and Gh_D05G2688, showed the opposite expression patterns, with the former being first down-regulated and then up-regulated, while the latter was down-regulated following up-regulation in the two lines. Over 40 genes encoding NB-ARC domain-containing disease resistance proteins were identified, while only the relatively highly expressed Gh_D11G3200 was first up-regulated and then down-regulated in the two lines.

In addition to the above-mentioned R-related genes, there were many other resistance genes involved in PTI and ETI. Two genes annotated as ADR1-like 1, namely, Gh_A05G1718 and Gh_D01G1013, were relatively highly expressed, with the former showing continuous up-regulation in the two lines, while the latter was down-regulated in CCRI36 and up-regulated following down-regulation in MBI8255. In the two lines, one MOS2 gene (Gh_A05G2156) was persistently down-regulated, while two ZED1 genes, Gh_A12G0580 and Gh_D12G0592, were up-regulated. Four MLP-like genes with high-level expression, Gh_D02G1410, Gh_D02G 1446, Gh_D07G2407, and Gh_D02G0560, were investigated, with the former two being up-regulated in CCRI36 and first up-regulated and then down-regulated in MBI8255, while the latter two showed up-regulation following down-regulation in the two lines. Both pathogenesis-related 4 (Gh_D11G2592) and PYR1-like 2 
(Gh_A04G0244) were up-regulated in the two lines, and the same pattern was observed in the three genes encoding P-loop containing nucleoside triphosphate hydrolases superfamily proteins (Gh_A12G1852, Gh_A1 2G1867, and Gh_D12G2022) in CCRI36, with opposite expressions observed between the former and the latter two. The RPS5-like 1 gene, Gh_A11G2559, was up-regulated in CCRI36, but was first up-regulated and then down-regulated in MBI8255, as observed in one highly expressed MLO gene, Gh_D06G2377. The same expression patterns were identified between other high-level MLO genes (Gh_D02G0670) and TAO1 (Gh_D06G2323), which showed up-regulation following down-regulation in the two lines. Interestingly, higherlevel expression was observed in the resistant lines over those in the susceptible line at 0 and 1 DAI, although diverse expression patterns were identified in the above immunity-related genes, which suggested that the greater resistance to VW might result from the better defense mechanisms in cotton.

\section{Conclusion}

In this study, artificial greenhouse, natural field, and disease nursery tests were conducted to confirm the selected CSSL (MBI8255) and its recurrent parent (CCRI36) together with two controls (Zhongzhimian2 and Jimian11), resulting in VW-resistant MBI8255 and VW-susceptible CCRI36. Subsequently, the two lines were separately subjected to biochemical tests and RNA-Seq, using root samples collected during the preliminary stages of V991 infection $(0,1$, and 2 DAI). In terms of the biochemical tests, the activities of POD and PPO were positively correlated with VW resistance, while a negative correlation was detected between MDA content and VW resistance. Additionally, a total of 77,412 genes, including 6934 novel genes, were identified in our RNA-Seq data, further leading to the identification of 23,180 DEGs through multiple comparisons. Following gene temporal expression pattern analysis, two identical profiles $(P$-value $\leq 0.05)$ derived from the DEGs of the two lines were further investigated by GO and KEGG enrichment analysis, which mostly clustered into metabolic process and cellular process in biological process, binding and catalytic activity in molecular function, and cell part and cell in cellular component, while significantly participating in the pathways of phenylpropanoid biosynthesis and plant hormone signal transduction. Finally, expression profiling of the DEGs related to the phenylpropanoid metabolic pathway, oxidation-reduction process, and plant resistance was carried out to identify the key candidate genes or pathways, which provided an abundance of information for revealing the mechanisms of VW resistance. The results of this study facilitate further research into cotton VW resistance by not only providing resistant material for cotton breeding, but also by improving our understanding of the mechanisms of VW resistance.

\section{Methods}

Preparation of $V$. dahliae and plant material

The $V$. dahliae fungus isolate used in this study was the highly aggressive defoliating V991. V991 was first cultured on solid potato dextrose agar (PDA) medium for 7 $\mathrm{d}$ at $25^{\circ} \mathrm{C}$ and then transferred to Czapek liquid medium to obtain conidia, after which it was placed in an incubated shaker (Haerbin Donglian Electonics, China) with a shaking speed of $150 \mathrm{rpm} / \mathrm{min}$ in darkness for 7-14 d at $25^{\circ} \mathrm{C}$. The concentration of the spore suspension was diluted to about $1 \times 10^{7}$ spores $/ \mathrm{mL}$ with sterile water for the root inoculation, which was assessed using a hemocytometer $[6,16]$.

The CSSLs constructed by our lab were derived from the hybridization between CCRI36 and Hai 1 in 2003 at the Institute of Cotton Research of the Chinese Academy of Agriculture Sciences (CRICAAS; Anyang, Henan province, China), in which the population was successfully developed through multiple generations of backcrossing and selfing, as described elsewhere [82]. CCRI36 developed by CRICAAS is a G. hirsutum cultivar with wide planting areas and high-yield fiber products, while Hai 1 stored by CRICAAS is a G. barbadense cultivar with excellent fiber quality and high resistance to Verticillium wilt [24]. Based on VW resistance investigations in multiple locations for years, MBI8255 and CCRI36 were found to be stably resistant and susceptible to VW, respectively. Two controls, Zhongzhimian2 and Jimain11, were selected as VW-resistant and VW-susceptible controls during the subsequent greenhouse and field tests.

\section{Greenhouse tests}

MBI8255 and CCRI36 together with the two controls (Zhongzhimian2 and Jimian11) were selected for the VW resistance tests in an artificial greenhouse located at the Institute of Cotton Research, Chinese Academy of Agricultural Sciences (ICR-CAAS). After disinfecting with $75 \%$ ethanol for $30 \mathrm{~s}$ and $2.5 \%$ sodium hypochlorite for $10 \mathrm{~min}$, the seeds were washed with sterile water 3-5 times and then subsequently placed into paper cups filled with sterilized sand and vermiculite in a proportion of 4:6. There were 7-10 seeds that were planted in each cup for germination, and 5 seedlings per cup that exhibited good and synchronous growth were reserved for further $V$. dahliae infection. Three replications were performed: (1) five paper cups from March 28, 2015 to May 30, 2015; (2) seven paper cups from August 26, 2015 to October 28, 2015; and (3) 10 paper cups from August 28, 2015 to October 30, 2015. When the first true leaf was open and flat, each paper cup was placed in a paper 
tray, the bottom of which had been removed with scissors, together with $10 \mathrm{~mL}$ of $1 \times 10^{7}$ spores $/ \mathrm{mL}$ conidial suspension.

Phenotypic identification of VW resistance was separately carried out at 15 and $30 \mathrm{DAI}$, and each seedling was evaluated according to a severity rating system (from 0 to 4) based on the disease symptoms of the plant leaves [83]. Specifically, 0 indicates healthy plant without disease symptom; 1 indicates less than $25 \%$ leaves with disease symptoms; 2 indicates $25-50 \%$ leaves with disease symptoms; 3 indicates $50-75 \%$ leaves with disease symptoms; and 4 indicates over $75 \%$ leaves with disease symptoms and even completely defoliated or dead plants. The DI was adopted to calculate the VW resistance using the following formula:

$$
\mathrm{DI}=\left[\sum(\mathrm{Ni} \times \mathrm{i}) /(\mathrm{N} \times 4)\right] \times 100
$$

where $\mathrm{i}$ is the disease grade from 0 to $4, \mathrm{Ni}$ is the number of plants with the corresponding disease grade, and $\mathrm{N}$ is the number of total plants investigated for each material.

\section{Natural field and disease nursery tests}

The natural field investigations of MBI8255, CCRI36, and the two controls were conducted on two different experimental farms: one replication in Anyang (Henan Province) and one replication in Shihezi (Xinjiang Province). Under natural conditions, the materials planted in the field for VW resistance investigations were infected with a mixture of $V$. dahliae isolates. The phenotypic identification of VW resistance was performed at the flowering and maturity stage in 2015 , and DI was calculated using the above-mentioned formula.

With the help of Professor Cai Yingfan, the disease nursery tests of MBI8255, CCRI36, and Jimian11 were conducted by the State Key Laboratory of Cotton Biology, Henan Key Laboratory of Plant Stress Biology, School of Life Science, Henan University. The phenotypic data were collected at the maturity stage in 2015, and DI was calculated using the formula described above.

\section{Sample collection}

Based on the VW-resistance results of the artificial greenhouse, natural field, and disease nursery tests, MBI8255 and CCRI36 were separately selected as the VW-resistant and VW-susceptible lines for further RNA-Seq and biochemical tests. The sterilized seeds were planted in three plastic trays filled with sterilized sand and vermiculite (4:6) and placed in a constant temperature incubator, with three biological replications. The culture conditions were set as follows: $25^{\circ} \mathrm{C}, 60$ $70 \%$ relative humidity, and a photoperiod of $14 \mathrm{~h} / 10 \mathrm{~h}$ (day/night). When the first true leaf was open and flat, the seedlings were removed from the plastic trays and inoculated via root-dipping into either the $1 \times 10^{7}$ spores $/ \mathrm{mL}$ conidia suspension or sterile water (for mock inoculation). After inoculation for $60 \mathrm{~s}$, the seedlings were transferred back to the plastic trays in the constant temperature incubator. The root samples were harvested at 0,1 , and 2 DAI, quickly frozen in liquid nitrogen, stored at $-80^{\circ} \mathrm{C}$, and divided into two portions: one for RNA-Seq with three replications and another for the biochemical tests with three replications.

\section{Biochemical tests}

Protective enzymes relevant to VW resistance were selected to investigate the activity changes during the preliminary stages of V991 infection (0, 1, and 2 DAI), including CAT, SOD, and POD. Fresh roots $(0.1 \mathrm{~g})$ per sample were ground in liquid nitrogen and suspended in $0.9 \mathrm{~mL}$ phosphate buffer ( $\mathrm{pH}$ 7.4) or saline solution. The $10 \%$ tissue homogenate of the CAT samples was centrifuged at $2500 \mathrm{rpm}$ for $10 \mathrm{~min}$, while those of the POD and SOD samples were centrifuged at $3500 \mathrm{rpm}$ for 15 $\mathrm{min}$. The obtained supernatants were collected to determine the activities of CAT, POD, and SOD using commercially available kits from the Nanjing Jiancheng Bioengineering Institute (Nanjing, China), and were separately measured using a spectrophotometer at 405,420 , and $550 \mathrm{~nm}$.

The defensive enzymes relevant to VW resistance, namely, PAL and PPO, were subjected to activity tests at 0,1 , and 2 DAI. Fresh roots $(0.1 \mathrm{~g})$ per sample were ground in liquid nitrogen and subsequently suspended in $0.9 \mathrm{~mL}$ extracting solution on ice. The tissue homogenate of the PAL sample was centrifuged at $4{ }^{\circ} \mathrm{C}$ and $10,000 \mathrm{rpm}$ for $10 \mathrm{~min}$, while the PPO sample was centrifuged at $4{ }^{\circ} \mathrm{C}$ at $8000 \mathrm{rpm}$ for $10 \mathrm{~min}$. The obtained supernatants were further processed according to the instructions of the PAL and POD assay kits from the Nanjing Jiancheng Bioengineering Institute and were measured spectrophotometrically at 209 and $525 \mathrm{~nm}$.

Biochemical substances relevant to VW resistance, such as MDA, soluble sugar, soluble protein, and proline, were selected to assess the content changes during the V991 infection process. Fresh roots $(0.1 \mathrm{~g})$ were ground in liquid nitrogen and then suspended in $0.9 \mathrm{~mL}$ solution of phosphate buffer ( $\mathrm{pH}$ 7.4) or saline for the MDA, soluble protein, and proline determinations. The homogenate of the MDA samples was centrifuged at $2500 \mathrm{rpm}$ for $10 \mathrm{~min}$, while that of the soluble protein and proline samples were centrifuged at $3500 \mathrm{rpm}$ for $15 \mathrm{~min}$. With regards to the content of soluble sugar, $0.1-0.2 \mathrm{~g}$ fresh roots were ground with $1 \mathrm{~mL}$ distilled water and then cultured in a boiling water bath for 10 min. After cooling, the tissue homogenate of the soluble 
sugar was centrifuged at room temperature at $8000 \mathrm{rpm}$ for $10 \mathrm{~min}$. The collected supernatants were further used for the content tests according to the instructions of the MDA, soluble sugar, soluble protein, and proline assay kits from the Nanjing Jiancheng Bioengineering Institute, and were separately measured spectrophotometrically at 532, 620, 595, and $520 \mathrm{~nm}$. Three biological replications per sample were performed.

\section{RNA extraction, library construction, and transcriptome sequencing}

According to the instruction manual of the RNAprep Pure Plant Kit (Tiangen, Beijing, China), the total RNA per sample was extracted from the frozen roots. The extracted RNA was first subjected to degradation and contamination detection by $1 \%$ agarose gel electrophoresis and integrity assessment by the RNA Nano 6000 Assay Kit of the Bioanalyzer 2100 system (Agilent Technologies, CA, USA), subsequently being calibrated by the Qubit $^{\circ}$ RNA Assay Kit in a Qubit $^{\circ}$ 2.0 Fluorometer (Life Technologies, CA, USA). High-quality RNA $(3 \mu \mathrm{g})$ per sample was required for cDNA library construction following the manufacturer's recommendations of NEBNext $^{\circ}$ Ultra $^{\text {Tm }}$ RNA Library rep Kit for Illumina ${ }^{\circ}$ (NEB, USA), and index codes were added to attribute the sequences to each sample. After being purified from total RNA by poly-T oligo-attached magnetic beads, mRNA was fragmented into 150-200 nt RNA inserts, which were used to synthesize the first-strand cDNA by random hexamer primer and M-MuLV Reverse Transcriptase $\left(\right.$ RNase $\left.\mathrm{H}^{-}\right)$and the second-strand cDNA by DNA Polymerase I and RNase $\mathrm{H}$. End-repair and adaptor ligation were performed on the double-stranded cDNA. The suitable fragments were purified with an AMPure XP system (Beckman Coulter, Beverly, USA) and enriched by PCR amplification. Subsequently, PCR products were purified using the AMPure XP system and assessment of library quality was performed on the Agilent Bioanalyzer 2100 system. For cluster generation, a cBot Cluster Generation System was used for clustering of the index-coded samples by the TruSeq Cluster Kit v3-cBot-HS (Illumina). In total, 18 cDNA libraries constructed from 18 RNA-Seq samples (two cotton lines at 0,1 , and 2 DAI with three replications) and were sequenced on a flow cell with an Illumina HiSeq 2500 sequencing platform.

\section{Data quality control and read mapping to the reference genome}

The raw data in FASTQ format were first processed through in-house Perl scripts, resulting in read sequences and their corresponding base qualities. The clean data were obtained by filtering adapters and low-quality reads with poly- $N>10 \%$ or $\mathrm{Q} 20<20 \%$ according to the Illumina pipeline, and the Q30 and GC content were subsequently calculated.

The high-quality clean reads were mapped to G. hirsutum as the reference genome [40] together with gene model annotation files, which were downloaded from the CottonGen database (http://www.cottongen.org). The index of the G. hirsutum genome was built by Bowtie v2.2.3 and the paired-end clean reads were aligned to the reference genome ( $G$. hirsutum) using TopHat v2.0.12 [84].

\section{DEG analysis}

In order to quantify the gene expression levels, the read numbers mapped to each gene were counted by HTSeq v0.6.1, and then the FPKM value was employed to calculate the expression level of each gene based on the length of the gene and the read counts mapped to this gene. As the most commonly utilized method for estimating gene expression levels at present, FPKM considers the effect of sequencing depth and gene length for the read counts at the same time [70].

The DESeq $\mathrm{R}$ package (v1.18.0) was used to identify differential gene expression between the control and treatment, which provided statistical routines for identifying the differential expression in the digital gene expression data using a model based on the negative binomial distribution. To manage the FDR, Benjamini and Hochberg's approach was performed to adjust the obtained $P$-values. DEGs were set as those genes with an adjusted $P$-value $\leq 0.01$ as determined by DESeq.

GO enrichment analysis of the DEGs was performed by the GOseq $\mathrm{R}$ package with a corrected $P$-value $\leq 0.5$ as the cutoff. KOBAS software was used to test the statistical enrichment of the DEGs in the KEGG pathways.

\section{Comparison of the expression patterns of the DEGs}

STEM software (Carnegie Mellon University, USA) was used to identify the temporal expression patterns of the DEGs in response to V991 infection (0, 1, and 2 DAI). GO and KEGG enrichment analysis was also employed to identify the potential functional genes or to characterize the expression profiles identified by STEM analysis.

\section{Validation of RNA-Seq by qRT-PCR}

In order to verify the reliability of the transcriptome data, qRT-PCR was performed on 20 randomly selected DEGs with three biological and technical replicates for each sample. The Primer-BLAST online tool of NCBI was used to design the specific primers of the selected DEGs. The cDNAs were synthesized using a TranScript All-in-One First-Strand cDNA Synthesis SuperMix for qPCR Kit (Transgen Biotech, Beijing, China). qRT-PCR was performed following the protocol of TransStart Top Green qPCR SuperMix kit (Transgen Biotech, Beijing, 
China) on the ABI 7500 fast Real-Time PCR System (Applied Biosystems, USA). The $\beta$-Actin housekeeping gene was used as the reference to normalize the relative expression levels, with the following primer sequences: F: 5'-ATCCTCCGTCTTGACCTTG-3' and R: 5'-TGTC CGTCAGGCAACTCAT-3'. The qRT-PCR carried out in a $20 \mu \mathrm{L}$ system at the following conditions: one cycle of $94{ }^{\circ} \mathrm{C}$ for 30s; 40 cycles of $94{ }^{\circ} \mathrm{C}$ for $5 \mathrm{~s}, 60^{\circ} \mathrm{C}$ for $34 \mathrm{~s}$, and one cycle of $60^{\circ} \mathrm{C}$ for $60 \mathrm{~s}$. The relative gene expression level was quantified using the $2^{-\Delta \Delta C t}$ method [85].

\section{Additional files}

Additional file 1: Differentially expressed genes between the samples. (XLS 3625 kb)

Additional file 2: Temporal expression of genes in two materials. (XLS $1123 \mathrm{~kb}$ )

Additional file 3: Gene names and their expression levels related with phenylopropanoid metabolic pathway. (XLS 20 kb)

\section{Abbreviations}

ADR1: Activated disease resistance 1; AOX: Alternative oxidase; APX: Ascorbate peroxidase; CAT: Cayalse; CSSLs: Chromosome Segment Substitution Lines; DAl: Days after inoculation; DEGs: Differentially expressed genes; DI: Disease index; ETI: Effector-triggered immunity; FDR: False discovery rate; FPKM: Fragments per kb per million of the mapped reads; GPX: Glutathione peroxidase; GRX: Glutaredoxins; HR: Hypersensitive response; MAS: Marker assisted-selection; MDA: Malondialdehyde; MDAR: Monodehydroascorbate reductase; MLO: Mildew locus O; MLP: Major latex protein; PAL: Phenylalanine ammonia-lyase; PAMPs: Pathogenassociated molecular patterns; PCC: Pearson Correlation Coefficient; PCD: Programmed cell death; PDA: Potato dextrose agar; POD: Peroxidase; PPO: Polyphenol oxidase; PRRs: Pattern recognition receptors; PrxR: Peroxiredoxin; PTI: PAMP-triggered immunity; qRT-PCR: Quantification real-time PCR; QTL: Quantitative trait locus; RBOHs: Respiratory burst oxidase homologs; ROS: Reactive oxygen species; RPS5: Resistance to pseudomonas syringae 5; SOD: Superoxide dismutase; STEM: Short Time-series Expression Mine STEM; TAO1: Target of AvrB operation 1; Tex: Thioredoxins; TTSS: Type III secretion system; WW: Verticillum wilt; ZED1: HOPZ-ACTIVARED RESISTANCE

\section{Acknowledgements}

Not applicable.

\section{Funding}

The construction of CSSL population in this study was supported by the National Natural Science Foundation of China (31101188), and the transcriptome sequencing and biochemical analysis were separately supported by the National Natural Science Foundation of China (31301343) and the Fundamental Research Funds for Central research institutes (Y2017JC48)

\section{Availability of data and materials}

The datasets generated and analysed during the current study are available from the corresponding author on reasonable request.

\section{Authors' contributions}

YLY, YZS and TTC conceived and designed the study. PTL and MH Rashid drafted the manuscript. AYL, JWG, JWL and HHS participated in the construction of CSSL population and trait identification in the field. QWL, QG, SQL and XHX did the resistance identification of Verticillium wilt in the greenhouse, natural field and disease nursery. QZ, LD, RXL and XYZ prepared the transcriptome samples and performed the RNA-seq analysis. ZZ, XJ, XYD and $Z Y$ participated in the biochemical tests and further analysis. YLY, WKG and RHP revised the manuscript. All the authors read and approved the final manuscript.
Ethics approval and consent to participate

Not applicable.

\section{Consent for publication}

Not applicable.

\section{Competing interests}

The authors declare that they have no competing interests.

\section{Publisher's Note}

Springer Nature remains neutral with regard to jurisdictional claims in published maps and institutional affiliations.

\section{Author details}

${ }^{1}$ State Key Laboratory of Cotton Biology, Key Laboratory of Biological and Genetic Breeding of Cotton, The Ministry of Agriculture, Institute of Cotton Research, Chinese Academy of Agricultural Science, Anyang 455000, Henan, China. ${ }^{2}$ School of Biotechnology and Food Engineering, Anyang Institute of Technology, Anyang 455000, Henan, China. ${ }^{3}$ College of Agriculture, South China Agricultural University, Guangzhou 510642, Guangdong, China.

Received: 7 December 2017 Accepted: 26 December 2018 Published online: 11 January 2019

\section{Reference}

1. Sunikunar G, Campbell LM, Puckhaber L, Stipanovic RD, Rsthore KS. Engineering cottonseed for use in human nutrition by tissue-specific reduction of toxic gossypol. Proc Natl Acad Sci U S A. 2006;104(48):18054-9.

2. Wendel JF, Albert VA. Phylogenetics of the cotton genus (Gossypium): character-state weighted parsimony analysis of chloroplast-DNA restriction site data and its systematic and biogeographic implications. Syst Bot. 1992; 17(1):115-43.

3. Wendel JF, Brubaker C, AlvaBmcrez I, Cronn R, Stewart JM, Paterson AH. Evolution and natural history of the cotton genus; 2009. p. 3-22.

4. Sink KC, Grey WE. A root-injection method to assess verticillium wilt resistance of peppermint ( Mentha $\times$ piperita L.) and its use in identifying resistant somaclones of cv. Black Mitcham. Euphytica. 1999:106(3):223-30.

5. Fang H, Zhou H, Sanogo S, Lipka AE, Fang DD, Percy RG, et al. Quantitative trait locus analysis of Verticillium wilt resistance in an introgressed recombinant inbred population of upland cotton. Mol Breed. 2014;33(3): 709-20.

6. Zhang J, Yu J, Pei W, Li X, Said J, Song M, et al. Genetic analysis of Verticillium wilt resistance in a backcross inbred line population and a meta-analysis of quantitative trait loci for disease resistance in cotton. BMC Genomics. 2015;16:577

7. Cai Y, Xiaohong H, Mo J, Sun Q, Yang J, Liu J. Molecular research and genetic engineering of resistance to Verticillium wilt in cotton: a review. Afr J Biotechnol. 2009:8(25):7363-72.

8. Bhat RG, Subbarao KV. Host range specificity in Verticillium dahliae. Phytopathology. 1999:89(12):1218-25.

9. Emilief F, Bartphj T. Physiology and molecular aspects of Verticillium wilt diseases caused by V. dahliae and V. albo-atrum. Mol Plant Pathol. 2006;7(2): $71-86$

10. Duressa D, Anchieta A, Chen D, Klimes A, Garciapedrajas MD, Dobinson KF, et al. RNA-seq analyses of gene expression in the microsclerotia of Verticillium dahliae. BMC Genomics. 2013;14(1):607.

11. Hu D, Wang C, Tao F, Cui O, Xu X, Shang W, et al. Whole genome wide expression profiles on germination of Verticillium dahliae microsclerotia. PLoS One. 2014:9(6):e100046.

12. Sun $Q$, Jiang $H$, Zhu X, Wang W, He X, Shi Y, et al. Analysis of sea-island cotton and upland cotton in response to Verticillium dahliae infection by RNA sequencing. BMC Genomics. 2013;14(1):852

13. Lüders RR, Galbieri R, Fuzatto MG, Cia E. Inheritance of resistance to Verticillium wilt in cotton. Crop BreedAppl Biotechnol. 2008;8(4):265-70.

14. Mert M, Kurt S, Gencer O, Akiscan Y, Boyaci K, Tok FM. Inheritance of resistance to Verticillium wilt (Verticillium dahliae) in cotton (Gossypium hirsutum L.). Plant Breed. 2005;124(1):102-4.

15. Palanga KK, Jamshed M, Rashid HO, Gong J, Li J, labal MS, et al. Quantitative trait locus mapping for Verticillium wilt resistance in an upland cotton recombinant inbred line using SNP-based high density genetic map. Front Plant Sci. 2017:8:382 
16. Wang $P$, Ning Z, Lin L, Chen H, Mei H, Zhao J, et al. Genetic dissection of tetraploid cotton resistant to Verticillium wilt using interspecific chromosome segment introgression lines. Crop J. 2014;2(5):278-88.

17. Eshed Y, Zamir D. An introgression line population of Lycopersicon pennellii in the cultivated tomato enables the identification and fine mapping of yield-associated QTL. Genetics. 1995;141(3):1147-62.

18. Liu S, Zhou R, Dong Y, Li P, Jia J. Development, utilization of introgression lines using a synthetic wheat as donor. Theor Appl Genet. 2006;112(7): 1360-73.

19. Takai T, Nonoue Y, Yamamoto SI, Yamanouchi U, Matsubara K, Liang ZW, et al. Development of chromosome segment substitution lines derived from backcross between indica donor rice cultivar 'Nona Bokra' and japonica recipient cultivar 'Koshihikari'. Breed Sci. 2007:57(3):257-61.

20. Zhu W, Lin J, Yang D, Zhao L, Zhang Y, Zhu Z, et al. Development of chromosome segment substitution lines derived from backcross between two sequenced rice cultivars indica recipient 93-11 and japonica donor nipponbare. Plant Mol Biol Report. 2009;27(2):126-31

21. Lacape JM, Llewellyn D, Jacobs J, Arioli T, Becker D, Calhoun S, et al. Meta-analysis of cotton fiber quality QTLs across diverse environments in a Gossypium hirsutum $\times$ G. barbadense RIL population. Bmc Plant Biol. 2010;10(1):132.

22. Yu JZ, Ulloa M, Hoffman SM, Kohel RJ, Pepper AE, Fang DD, et al. Mapping genomic loci for cotton plant architecture, yield components, and fiber properties in an interspecific (Gossypium hirsutum L. $\times$ G. barbadense L.) RIL population. Mol Genet Genomics Mgg. 2014;289(6):1347.

23. Said JI, Knapka JA, Song M, Zhang J. Cotton QTLdb: a cotton QTL database for QTL analysis, visualization, and comparison between Gossypium hirsutum and $G$. hirsutum $\times$ G. barbadense populations. Mol Genet Genomics. 2015;290(4):1615-25.

24. Shi Y, Zhang B, Liu A, Li W, Li J, Lu Q, et al. Quantitative trait loci analysis of Verticillium wilt resistance in interspecific backcross populations of Gossypium hirsutum × Gossypium barbadense. BMC Genomics. 2016;17(1):877.

25. Zhai H, Gong W, Tan Y, Liu A, Song W, Li J, et al. Identification of chromosome segment substitution lines of Gossypium barbadense introgressed in G. hirsutum and quantitative trait locus mapping for fiber quality and yield traits. Plos One. 2016;11(9):e0159101.

26. Zheng JY, Oluoch G, Riaz Khan MK, Wang XX, Cai XY, Zhou ZL, et al. Mapping QTLs for drought tolerance in an F2:3 population from an interspecific cross between Gossypium tomentosum and Gossypium hirsutum. Genet Mol Res Gmr. 2016:15(3).

27. Jones JD, Dangl JL. The plant immune system. Nature. 2006;444(7117):323.

28. Dodds PN, Rathjen JP. Plant immunity: towards an integrated view of plantpathogen interactions. Nat Rev Genet. 2010;11(8):539-48.

29. Lahaye T, Bonas U. Molecular secrets of bacterial type III effector proteins. Trends Plant Sci. 2001;6(10):479-85.

30. Lam E, Kato N, Lawton M. Programmed cell death, mitochondria and the plant hypersensitive response. Nature. 2001;411(6839):848-53.

31. Shirasu K, Schulze-Lefert P. Regulators of cell death in disease resistance. Plant Mol Biol. 2000:44(3):371-85.

32. Martin GB, Bogdanove AJ, Sessa G. Understanding the functions of plant disease resistance proteins. Annu Rev Plant Biol. 2003;54:23-61.

33. Takken FL, Albrecht M, Tameling Wl. Resistance proteins: molecular switches of plant defence. Curr Opin Plant Biol. 2006;9(4):383-90.

34. Luck JE, Lawrence GJ, Dodds PN, Shepherd KW, Ellis JG. Regions outside of the leucine-rich repeats of flax rust resistance proteins play a role in specificity determination. Plant Cell. 2000;12(8):1367-77.

35. Cannon SB, Zhu H, Baumgarten AM, Spangler R, May G, Cook DR, et al. Diversity, distribution, and ancient taxonomic relationships within the TIR and non-TIR NBS-LRR resistance gene subfamilies. J Mol Evol. 2002;54(4): 548-62.

36. Meyers BC, Dickerman AW, Michelmore RW, Sivaramakrishnan S, Sobral BW, Young ND. Plant disease resistance genes encode members of an ancient and diverse protein family within the nucleotide-binding superfamily. Plant J. 1999:20(3):317-32

37. Wang K, Wang Z, Li F, Ye W, Wang J, Song G, et al. The draft genome of a diploid cotton Gossypium raimondii. Nat Genet. 2012;44(10):1098-103.

38. Li F, Fan G, Wang K, Sun F, Yuan Y, Song G, et al. Genome sequence of the cultivated cotton Gossypium arboreum. Nat Genet. 2014;46(6):567-72.

39. Li F, Fan G, Lu C, Xiao G, Zou C, Kohel RJ, et al. Genome sequence of cultivated upland cotton (Gossypium hirsutum TM-1) provides insights into genome evolution. Nat Biotechnol. 2015;33(5):524-30.
40. Zhang T, Hu Y, Jiang W, Fang L, Guan X, Chen J, et al. Sequencing of allotetraploid cotton (Gossypium hirsutum L. acc. TM-1) provides a resource for fiber improvement. Nat Biotechnol. 2015;33(5):531-7.

41. Yuan D, Tang Z, Wang M, Gao W, Tu L, Xin J, et al. The genome sequence of Sea-Island cotton (Gossypium barbadense) provides insights into the allopolyploidization and development of superior spinnable fibres. Sci Rep. 2015;5(6):17662.

42. Liu X, Zhao B, Zheng HJ, Hu Y, Lu G, Yang CQ, et al. Gossypium barbadense genome sequence provides insight into the evolution of extra-long staple fiber and specialized metabolites. Sci Rep. 2015;5:14139.

43. $\mathrm{Xu} \mathrm{L}$, Zhu L, Tu L, Liu L, Yuan D, Jin L, et al. Lignin metabolism has a central role in the resistance of cotton to the wilt fungus Verticillium dahliae as revealed by RNA-Seq-dependent transcriptional analysis and histochemistry. J Exp Bot. 2011;62(15):5607-21.

44. Faino $\mathrm{L}$, de Jonge $\mathrm{R}$, Thomma BP. The transcriptome of Verticillium dahliaeinfected Nicotiana benthamiana determined by deep RNA sequencing. Plant Signal Behav. 2012;7(9):1065-9.

45. Xiong D, Wang Y, Tian C. Transcriptomic profiles of the smoke tree wilt fungus Verticillium dahliae under nutrient starvation stresses. Mol Gen Genomics. 2015;290(5):1963-77.

46. Fu X, Wu X, Zhou X, Liu S, Shen Y, Wu F. Companion cropping with potato onion enhances the disease resistance of tomato against Verticillium dahliae Front Plant Sci. 2015;6:726.

47. Guo S, Zuo Y, Zhang Y, Wu C, Su W, Jin W, et al. Large-scale transcriptome comparison of sunflower genes responsive to Verticillium dahliae. BMC Genomics. 2017;18(1):42.

48. Jimenez-Ruiz J, Leyva-Perez MO, Schiliro E, Barroso JB, Bombarely A, Mueller $L$, et al. Transcriptomic analysis of $L$. roots during the early infection process. Plant Genome. 2017;10(1).

49. Lu Q, Shi Y, Xiao X, Li P, Gong J, Gong W, et al. Transcriptome analysis suggests that chromosome introgression fragments from sea island cotton (Gossypium barbadense) increase fiber strength in upland cotton (Gossypium hirsutum). G3 (Bethesda). 2017;7(10):3469-79.

50. $\mathrm{Ma} \mathrm{QH}$, Zhu HH, Han JQ. Wheat ROP proteins modulate defense response through lignin metabolism. Plant Sci. 2017;262:32-8.

51. Dickinson BC, Chang CJ. Chemistry and biology of reactive oxygen species in signaling or stress responses. Nat Chem Biol. 2011;7(8):504-11.

52. Marino D, Dunand C, Puppo A, Pauly N. A burst of plant NADPH oxidases. Trends Plant Sci. 2012;17(1):9-15

53. Bonardi V, Tang S, Stallmann A, Roberts M, Cherkis K, Dangl JL. Expanded functions for a family of plant intracellular immune receptors beyond specific recognition of pathogen effectors. Proc Natl Acad Sci U S A. 2011; 108(39):16463-8

54. Zhang Y, Cheng YT, Bi D, Palma K, Li X. MOS2, a protein containing G-patch and KOW motifs, is essential for innate immunity in Arabidopsis thaliana. Curr Biol. 2005;15(21):1936-42.

55. Wang Z, Cui D, Liu J, Zhao J, Liu C, Xin W, et al. Arabidopsis ZED1-related kinases mediate the temperature-sensitive intersection of immune response and growth homeostasis. New Phytol. 2017:215(2):711-24.

56. Radauer $C$, Lackner $P$, Breiteneder $H$. The bet $\vee 1$ fold: an ancient, versatile scaffold for binding of large, hydrophobic ligands. BMC Evol Biol. 2008:8:286.

57. Sels J, Mathys J, De Coninck BM, Cammue BP, De Bolle MF. Plant pathogenesis-related (PR) proteins: a focus on PR peptides. Plant Physiol Biochem. 2008;46(11):941-50.

58. Zheng X, Chen X, Zhang X, Lin Z, Shang J, Xu J, et al. Isolation and identification of a gene in response to rice blast disease in rice. Plant Mol Biol. 2004;54(1):99-109.

59. Lievens L, Pollier J, Goossens A, Beyaert R, Staal J. Abscisic acid as pathogen effector and immune regulator. Front Plant Sci. 2017;8:587.

60. Simonich MT, Innes RW. A disease resistance gene in Arabidopsis with specificity for the avrPph3 gene of Pseudomonas syringae pv. Phaseolicola. Mol Plant-Microbe Interact. 1995:8(4):637-40.

61. Qi D, DeYoung BJ, Innes RW. Structure-function analysis of the coiled-coil and leucine-rich repeat domains of the RPS5 disease resistance protein. Plant Physiol. 2012;158(4):1819-32.

62. Piffanelli $P$, Zhou F, Casais C, Orme J, Jarosch B, Schaffrath U, et al. The barley MLO modulator of defense and cell death is responsive to biotic and abiotic stress stimuli. Plant Physiol. 2002;129(3):1076-85.

63. Eitas TK, Nimchuk ZL, Dangl JL. Arabidopsis TAO1 is a TIR-NB-LRR protein that contributes to disease resistance induced by the Pseudomonas syringae effector AvrB. Proc Natl Acad Sci U S A. 2008;105(17):6475-80. 
64. BEJARANO-ALCÁZAR J, BLANCO-LÓPEZ MA, MELERO-VARA JM, JIMÉNEZDÍAZ RM. The influence of Verticillium wilt epidemics on cotton yield in southern Spain. Plant Pathol. 2010;46(2):168-78.

65. Mittler R, Vanderauwera S, Gollery M, Van Breusegem F. Reactive oxygen gene network of plants. Trends Plant Sci. 2004;9(10):490-8.

66. Mauch-Mani B, Slusarenko AJ. Production of salicylic acid precursors is a major function of phenylalanine ammonia-lyase in the resistance of Arabidopsis to Peronospora parasitica. Plant Cell. 1996;8(2):203-12.

67. Zhou B. Correlation between resistance of eggplant and defense-related enzymes and biochemical substances of leaves. Afr J Biotechnol. 2012; 11(74):13896-902.

68. Chaoui A, Mazhoudi S, Ghorbal MH, Ferjani EE. Cadmium and zinc induction of lipid peroxidation and effects on antioxidant enzyme activities in bean ( Phaseolus vulgaris L.). Plant Sci. 1997;127(2):139-47.

69. Radwan DEM, Fayez KA, Mahmoud SY, Hamad A, Lu G. Salicylic acid alleviates growth inhibition and oxidative stress caused by zucchini yellow mosaic virus infection in Cucurbita pepo leaves. Physiol Mol Plant Pathol. 2006;69(4):172-81.

70. Trapnell C, Williams BA, Pertea G, Mortazavi A, Kwan G, van Baren MJ, et al. Transcript assembly and quantification by RNA-Seq reveals unannotated transcripts and isoform switching during cell differentiation. Nat Biotechnol. 2010:28(5):511-5.

71. Suriyan C, Chalermpol K. Effects of water stress induced by sodium chloride and mannitol on proline accumulation, photosynthetic abilities and growth characters of eucalyptus (Eucalyptus camaldulensis Dehnh.). New For. 2010; 40(3):349-60.

72. Bell AA. Phytoalexin production and Verticillium wilt resistance in cotton. Tech Rep Arch Image Library. 1969;2013(8):1-8.

73. Chen F, Liu CJ, Tschaplinski TJ, Zhao N, Gray DJ, Trigiano RN. Genomics of secondary metabolism in Populus: interactions with biotic and abiotic environments. Crit Rev Plant Sci. 2009;28(5):375-92.

74. Bartwal A, Mall R, Lohani P, Guru SK, Arora S. Role of secondary metabolites and brassinosteroids in plant defense against environmental stresses. J Plant Growth Regul. 2013;32(1):216-32.

75. Vanholme R, Morreel K, Darrah C, Oyarce P, Grabber JH, Ralph J, et al. Metabolic engineering of novel lignin in biomass crops. New Phytol. 2012; 196(4):978-1000

76. Cabane M, Afif D, Hawkins S. Lignins and abiotic stresses. Adv Bot Res. 2016; 61:219-62.

77. Li Q, Song J, Peng S, Wang JP, Qu GZ, Sederoff RR, et al. Plant biotechnology for lignocellulosic biofuel production. Plant Biotechnol J. 2015;12(9):1174-92.

78. Wang W, Li R, Zhu Q, Tang X, Zhao Q. Transcriptomic and physiological analysis of common duckweed Lemna minor responses to $\mathrm{NH} 4(+)$ toxicity. BmcPlant Biol. 2016;16:92.

79. Apel K, Hirt H. Reactive oxygen species: metabolism, oxidative stress, and signal transduction. Annu Rev Plant Biol. 2004;55(x):373-99.

80. Clay NK, Adio AM, Denoux C, Jander G, Ausubel FM. Glucosinolate metabolites required for an Arabidopsis innate immune response. Science. 2009:323(5910):95-101.

81. Kim KH, Kang YJ, Kim DH, Yoon MY, Moon JK, Kim MY, et al. RNA-Seq analysis of a soybean near-isogenic line carrying bacterial leaf pustuleresistant and -susceptible alleles. DNA Res. 2011;18(6):483-97.

82. Li PT, Wang M, Lu QW, Ge Q, Rashid M, Liu AY, et al. Comparative transcriptome analysis of cotton fiber development of Upland cotton (Gossypium hirsutum) and Chromosome Segment Substitution Lines from G. hirsutum $\times$ G. barbadense. Bmc Genomics. 2017;18(1):705.

83. Ning Z, Zhao R, Chen H, Ai N, Zhang X, Zhao J, et al. Molecular tagging of a major quantitative trait locus for broad-spectrum resistance to Verticillium wilt in upland cotton cultivar Prema. Crop Sci. 2013;53(6):2304-12.

84. Kim D, Pertea G, Trapnell C, Pimentel H, Kelley R, Salzberg SL. TopHat2: accurate alignment of transcriptomes in the presence of insertions, deletions and gene fusions. Genome Biol. 2013;14(4):R36.

85. Livak K, Schmittgen TD. Analysis of relative gene expression data using realtime quantitative PCR and the $2^{-\Delta \Delta \mathrm{ct}}$ method. Methods. 2012;25(4):402-8.

\section{Ready to submit your research? Choose BMC and benefit from:}

- fast, convenient online submission

- thorough peer review by experienced researchers in your field

- rapid publication on acceptance

- support for research data, including large and complex data types

- gold Open Access which fosters wider collaboration and increased citations

- maximum visibility for your research: over $100 \mathrm{M}$ website views per year

At BMC, research is always in progress.

Learn more biomedcentral.com/submissions 